\title{
Development of a Novel Route for Incorporation of Carbon-14 into the Pyridine Ring of Rinskor ${ }^{\mathrm{TM}}$ Active
}

\author{
Pete Johnson
}

Process Chemistry, Corteva Agriscience ${ }^{\mathrm{TM}}$, 9330 Zionsville Rd., Indianapolis, IN 46268, USA

\section{Supporting Information}

Table of Contents

Figure S1-a. ${ }^{1} \mathrm{H}$ NMR spectra $\left(\mathrm{CDCl}_{3}\right)$ of 2,2,2-trifluoro- $N$-tritylethanimidoyl chloride (17)............ 4

Figure S1-b. ${ }^{19} \mathrm{~F}$ NMR spectra $\left(\mathrm{CDCl}_{3}\right)$ of 2,2,2-trifluoro- $N$-tritylethanimidoyl chloride (17)............. 5

Figure S1-c. ${ }^{13} \mathrm{C}$ NMR spectra $\left(\mathrm{CDCl}_{3}\right)$ of 2,2,2-trifluoro- $N$-tritylethanimidoyl chloride (17). ........... 6

Figure S2-a. ${ }^{1} \mathrm{H}$ NMR spectra $\left(\mathrm{CDCl}_{3}\right)$ of $N$-[5,5-Diethoxy-1,1,1-trifluoropent-3-yn-2-ylidene]-1,1,1-

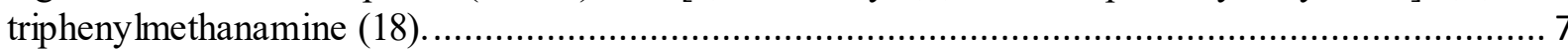

Figure S2-b. ${ }^{19} \mathrm{~F}$ NMR spectra $\left(\mathrm{CDCl}_{3}\right)$ of $N$-[5,5-Diethoxy-1,1,1-trifluoropent-3-yn-2-ylidene]-1,1,1-

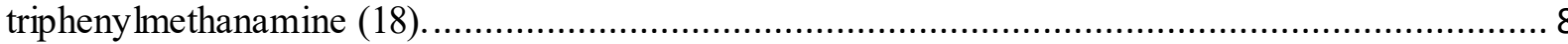

Figure S2-c. ${ }^{13} \mathrm{C}$ NMR spectra $\left(\mathrm{CDCl}_{3}\right)$ of $N$-[5,5-Diethoxy-1,1,1-trifluoropent-3-yn-2-ylidene]-1,1,1-

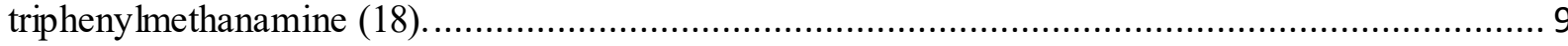

Figure S3-a. ${ }^{1} \mathrm{H}$ NMR spectra $\left(\mathrm{CDCl}_{3}\right)$ of 4-chloro-2-fluoro-3-methoxybenzaldehyde $O$-methyl oxime (20)

Figure S3-b. ${ }^{19} \mathrm{~F}$ NMR spectra $\left(\mathrm{CDCl}_{3}\right)$ of 4-chloro-2-fluoro-3-methoxybenzaldehyde $O$-methyl oxime (20)

Figure S3-c. ${ }^{13} \mathrm{C}$ NMR spectra $\left(\mathrm{CDCl}_{3}\right)$ of 4-chloro-2-fluoro-3-methoxybenza ldehyde $O$-methyl oxime (20).

Figure S4-a. ${ }^{1} \mathrm{H}$ NMR spectra $\left(\mathrm{CDCl}_{3}\right)$ of 1-(4-chloro-2-fluoro-3-methoxyphenyl)-methanamine (13). 13 Figure S4-b. ${ }^{19} \mathrm{~F}$ NMR spectra $\left(\mathrm{CDCl}_{3}\right)$ of 1-(4-chloro-2-fluoro-3-methoxyphenyl)-methanamine (13).

Figure S4-c. ${ }^{13} \mathrm{C} \mathrm{NMR}$ spectra $\left(\mathrm{CDCl}_{3}\right)$ of 1-(4-chloro-2-fluoro-3-methoxyphenyl)-methanamine (13). 
Figure S5-a. 1H NMR spectra (CDC13) of 2-(4-chloro-2-fluoro-3-methoxyphenyl)-6-(diethoxymethyl)3-fluoro-N-tritylpyridin-4-amine (21)..... 16

Figure S5-b. ${ }^{19} \mathrm{~F}$ NMR spectra $\left(\mathrm{CDCl}_{3}\right)$ of 2-(4-chloro-2-fluoro-3-methoxyphenyl)-6-(diethoxymethyl)3-fluoro- $N$-tritylpyridin-4-amine (21). 17

Figure S5-c. ${ }^{13} \mathrm{C}$ NMR spectra $\left(\mathrm{CDCl}_{3}\right)$ of 2-(4-chloro-2-fluoro-3-methoxyphenyl)-6-(diethoxymethyl)3-fluoro- $N$-tritylpyridin-4-amine (21). 18

Figure S6-a. ${ }^{1} \mathrm{H}$ NMR spectra (DMSO- $d_{6}$ ) of 4-amino-6-(4-chloro-2-fluoro-3-methoxyphenyl)-5-

fluoropyridine-2-carbaldehyde (22). 19

Figure S6-b. ${ }^{19} \mathrm{~F}$ NMR spectra (DMSO- $d_{6}$ ) of 4-amino-6-(4-chloro-2-fluoro-3-methoxyphenyl)-5fluoropyridine-2-carbaldehyde (22). 20

Figure S6-c. ${ }^{13} \mathrm{C}$ NMR spectra (DMSO- $d_{6}$ ) of 4-amino-6-(4-chloro-2-fluoro-3-methoxyphenyl)-5fluoropyridine-2-carbaldehyde (22).

Figure S7-a. 1H NMR spectra (DMSO- $d_{6}$ ) of 4-Amino-3-chloro-6-(4-chloro-2-fluoro-3methoxyphenyl)-5-fluoropyridine-2-carbaldehyde (23).

Figure S7-b. ${ }^{19} \mathrm{~F}$ NMR spectra (DMSO- $d_{6}$ ) of 4-Amino-3-chloro-6-(4-chloro-2-fluoro-3methoxyphenyl)-5-fluoropyridine-2-carbaldehyde (23). 23

Figure S7-c. ${ }^{13} \mathrm{C}$ NMR spectra (DMSO- $d_{6}$ ) of 4-Amino-3-chloro-6-(4-chloro-2-fluoro-3methoxyphenyl)-5-fluoropyridine-2-carbaldehyde (23).

Figure S8-a. ${ }^{1} \mathrm{H}$ NMR spectra (DMSO- $d_{6}$ ) of 4-amino-3-chloro-6-(4-chloro-2-fluoro-3methoxyphenyl)-5-fluoropyridine-2-carboxylic acid (24).

Figure S8-b. ${ }^{19} \mathrm{~F}$ NMR spectra (DMSO- $d_{6}$ ) of 4-amino-3-chloro-6-(4-chloro-2-fluoro-3methoxyphenyl)-5-fluoropyridine-2-carboxylic acid (24).

Figure S8-c. ${ }^{13} \mathrm{C}$ NMR spectra (DMSO- $d_{6}$ ) of 4-amino-3-chloro-6-(4-chloro-2-fluoro-3methoxyphenyl)-5-fluoropyridine-2-carboxylic acid (24).

Figure S9-a. ${ }^{1} \mathrm{H}$ NMR spectra $\left(\mathrm{CDCl}_{3}\right)$ of benzyl 4-amino-3-chloro-6-(4-chloro-2-fluoro-3methoxyphenyl)-5-fluoropyridine-2-carboxylate (Rinskor, 1).

Figure S9-b. ${ }^{19} \mathrm{~F}$ NMR spectra $\left(\mathrm{CDCl}_{3}\right)$ of benzyl 4-amino-3-chloro-6-(4-chloro-2-fluoro-3methoxyphenyl)-5-fluoropyridine-2-carboxylate (Rinskor, 1).

Figure S9-c. ${ }^{13} \mathrm{C}$ NMR spectra $\left(\mathrm{CDCl}_{3}\right)$ of benzyl 4-amino-3-chloro-6-(4-chloro-2-fluoro-3methoxyphenyl)-5-fluoropyridine-2-carboxylate (Rinskor, 1). 30

Figure S10. HPLC chromatograms for 2-(4-chloro-2-fluoro-3-methoxyphenyl)-6-(diethoxymethyl)-3fluoro- $N$-trityl $\left(4-{ }^{14} \mathrm{C}\right)$ pyridin-4-amine (26).

Figure S11. HPLC chromatograms for 4-Amino-6-(4-chloro-2-fluoro-3-methoxyphenyl)-5-fluoro(4-

$\left.{ }^{14} \mathrm{C}\right)$ pyridine-2-carbaldehyde (27).

Figure S12. HPLC chromatograms for 4-amino-3-chloro-6-(4-chloro-2-fluoro-3-methoxyphenyl)-5fluoro $\left(4-{ }^{14} \mathrm{C}\right)$ pyridine-2-carbaldehyde (28)...... 33

Figure S13. HPLC chromatograms for 4-amino-3-chloro-6-(4-chloro-2-fluoro-3-methoxyphenyl)-5fluoro $\left(4-{ }^{14} \mathrm{C}\right)$ pyridine-2-carboxylic acid (29). 
Figure S14. HPLC chromatograms for benzyl 4-amino-3-chloro-6-(4-chloro-2-fluoro-3methoxyphenyl)-5-fluoro $\left(4-{ }^{14} \mathrm{C}\right)$ pyridine-2-carboxylate $\left(30\right.$, Rinskor-Py-4- $\left.{ }^{14} \mathrm{C}\right)$. 
Figure S1-a. ${ }^{1} \mathrm{H}$ NMR spectra $\left(\mathrm{CDCl}_{3}\right)$ of 2,2,2-trifluoro- $N$-tritylethanimidoyl chloride (17).

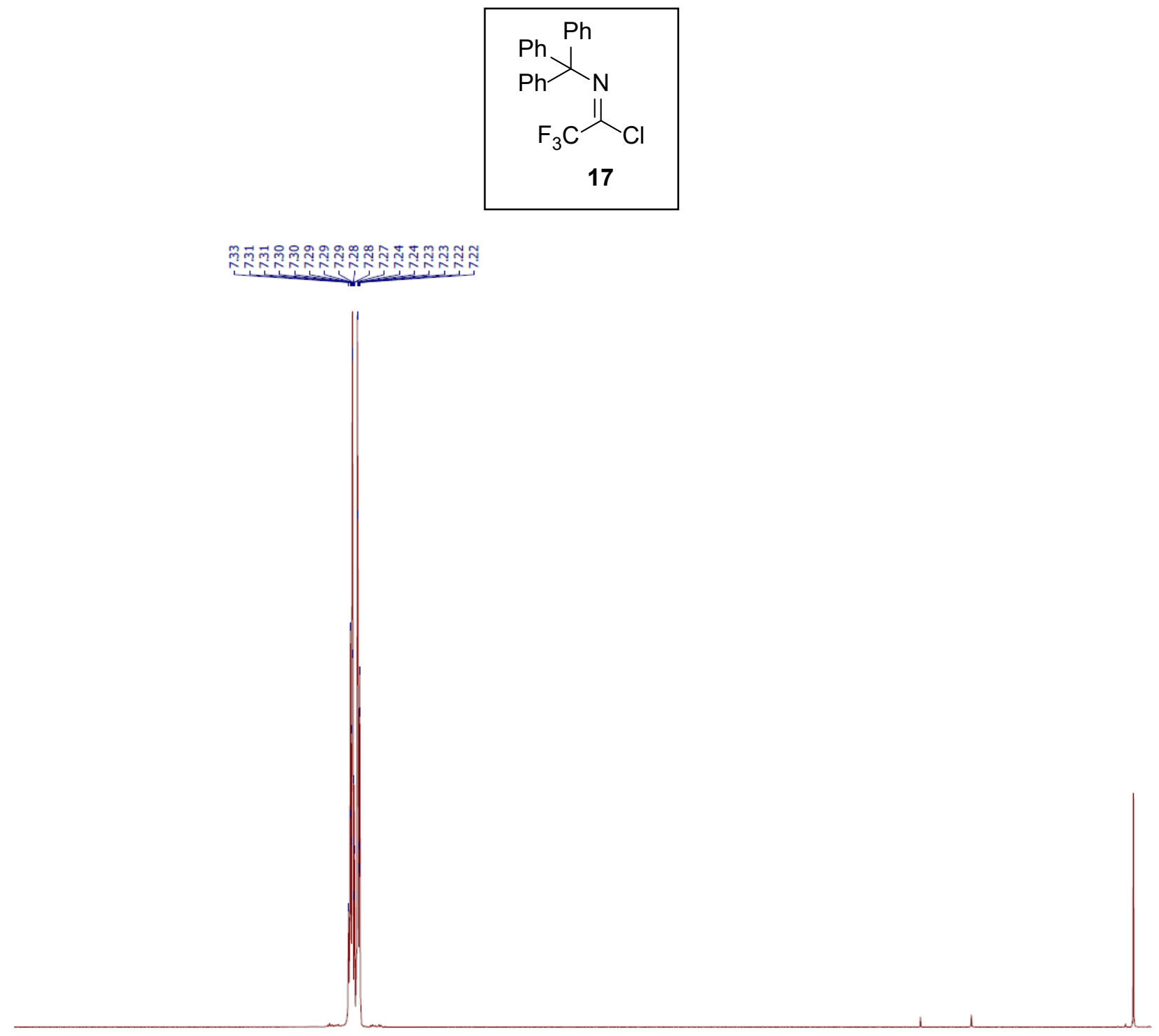

\begin{tabular}{llllllllllllllllllllllllllll}
\hline 5 & 10.0 & 9.5 & 9.0 & 8.5 & 8.0 & 7.5 & 7.0 & 6.5 & 6.0 & $\underset{f 1}{5}(\mathrm{nnm})$ & 5.0 & 4.5 & 4.0 & 3.5 & 3.0 & 2.5 & 2.0 & 1.5 & 1.0 & 0.5 & 0.0
\end{tabular} 
Figure S1-b. ${ }^{19} \mathrm{~F}$ NMR spectra $\left(\mathrm{CDCl}_{3}\right)$ of 2,2,2-trifluoro- $N$-tritylethanimidoyl chloride (17).

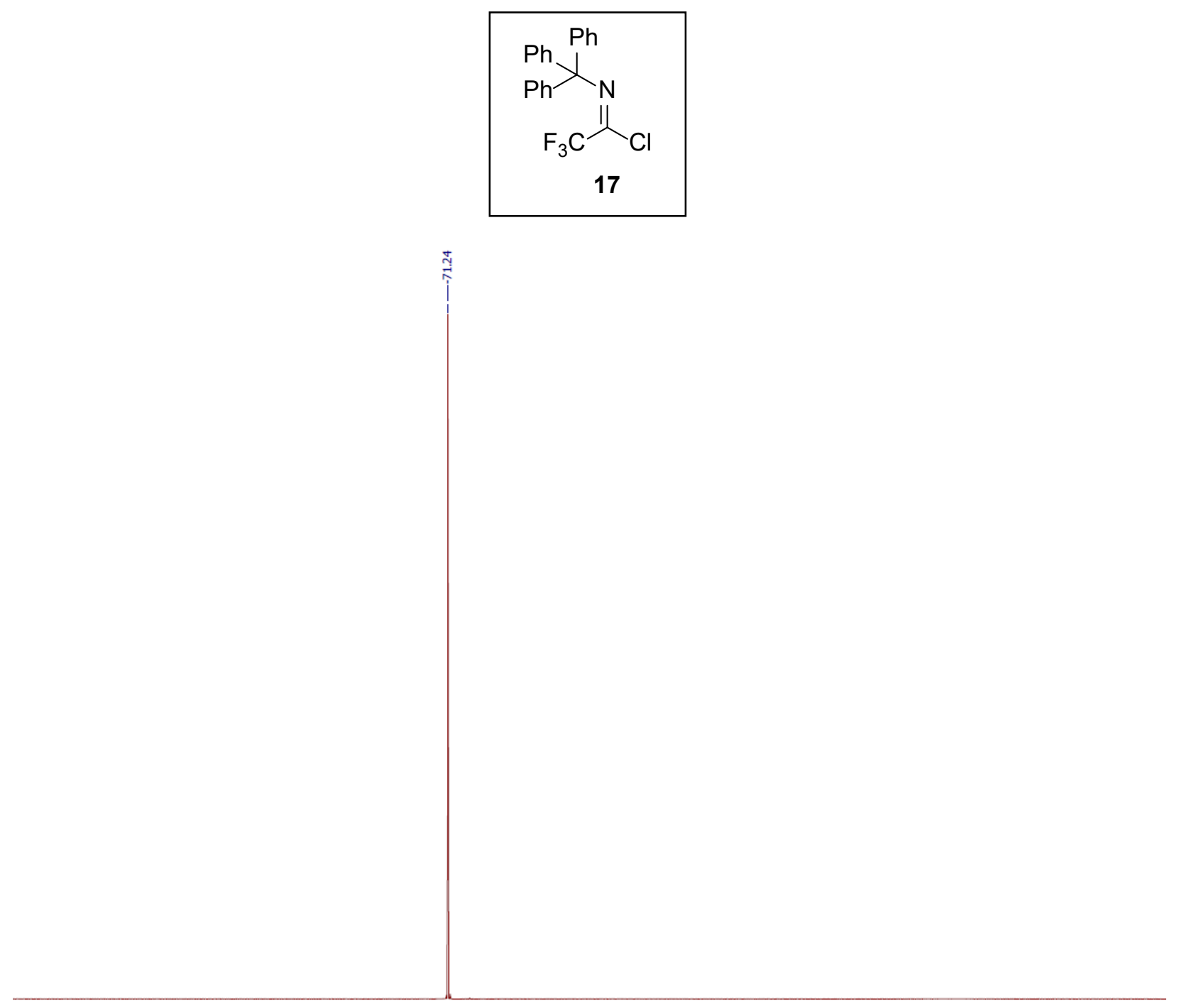

$\begin{array}{llllllllllllllllllllllllllllllllllll}10 & 0 & -10 & -20 & -30 & -40 & -50 & -60 & -70 & -80 & -90 & -100 & -110 & -120 & -130 & -140 & -150 & -160 & -170 & -180 & -190 & -200 & -210\end{array}$ 
Figure S1-c. ${ }^{13} \mathrm{C}$ NMR spectra $\left(\mathrm{CDCl}_{3}\right)$ of 2,2,2-trifluoro- $N$-tritylethanimidoyl chloride (17).

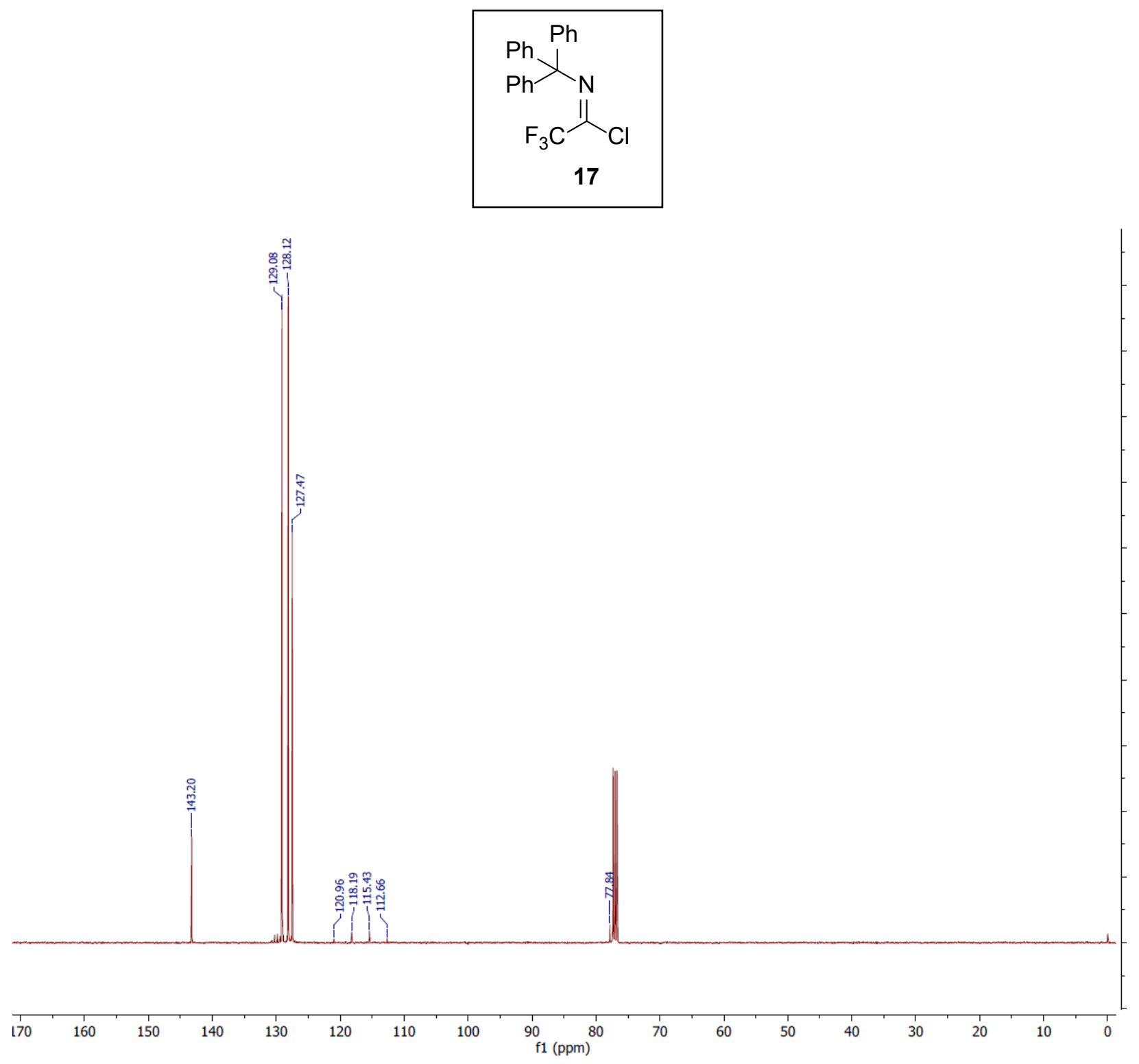


Figure S2-a. ${ }^{1} \mathrm{H}$ NMR spectra $\left(\mathrm{CDCl}_{3}\right)$ of $N$-[5,5-Diethoxy-1,1,1-trifluoropent-3-yn-2-ylidene]1,1,1-triphenylmethanamine (18).

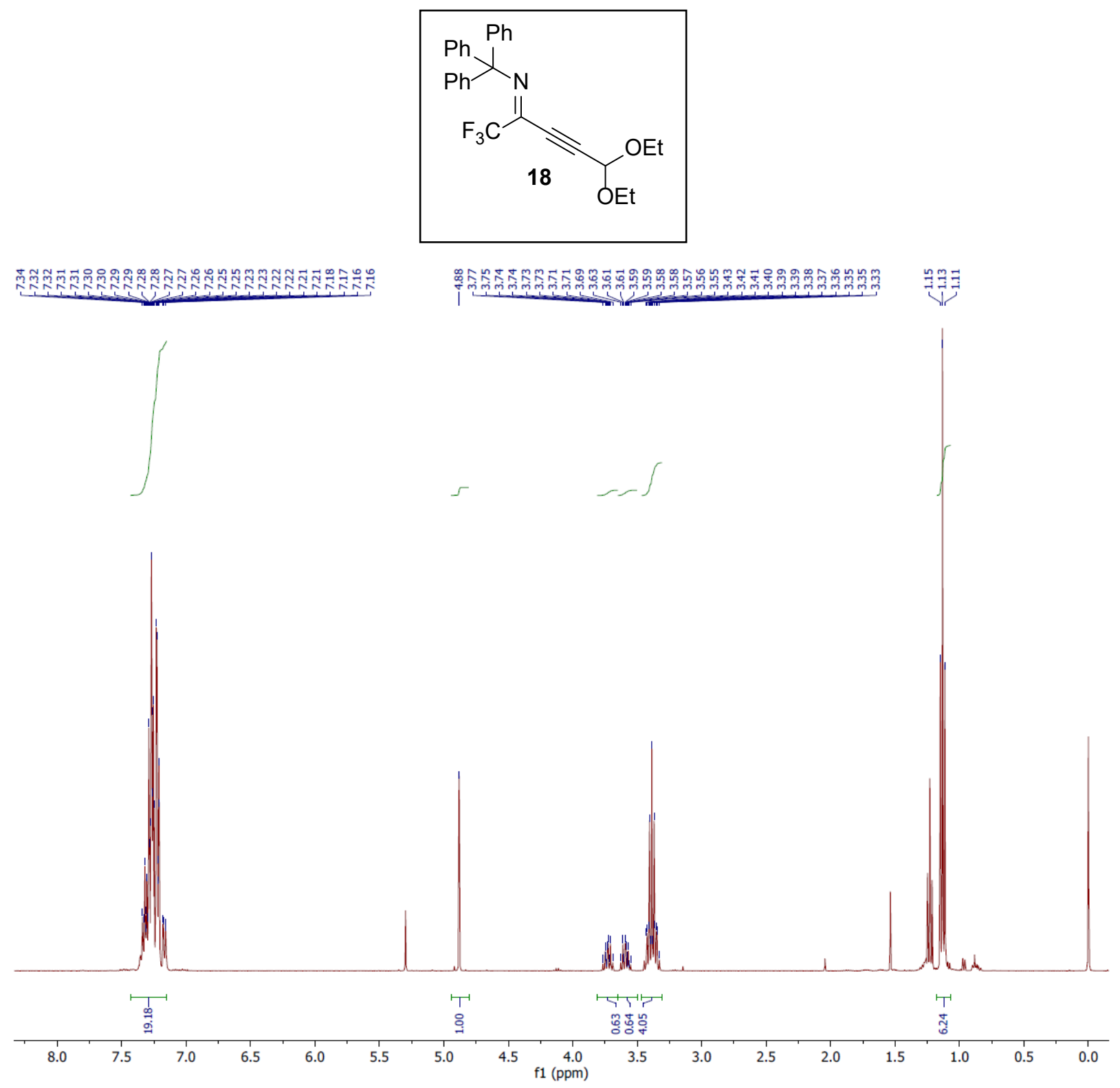


Figure S2-b. ${ }^{19} \mathrm{~F}$ NMR spectra $\left(\mathrm{CDCl}_{3}\right)$ of $N$-[5,5-Diethoxy-1,1,1-trifluoropent-3-yn-2-ylidene]1,1,1-triphenylmethanamine (18).
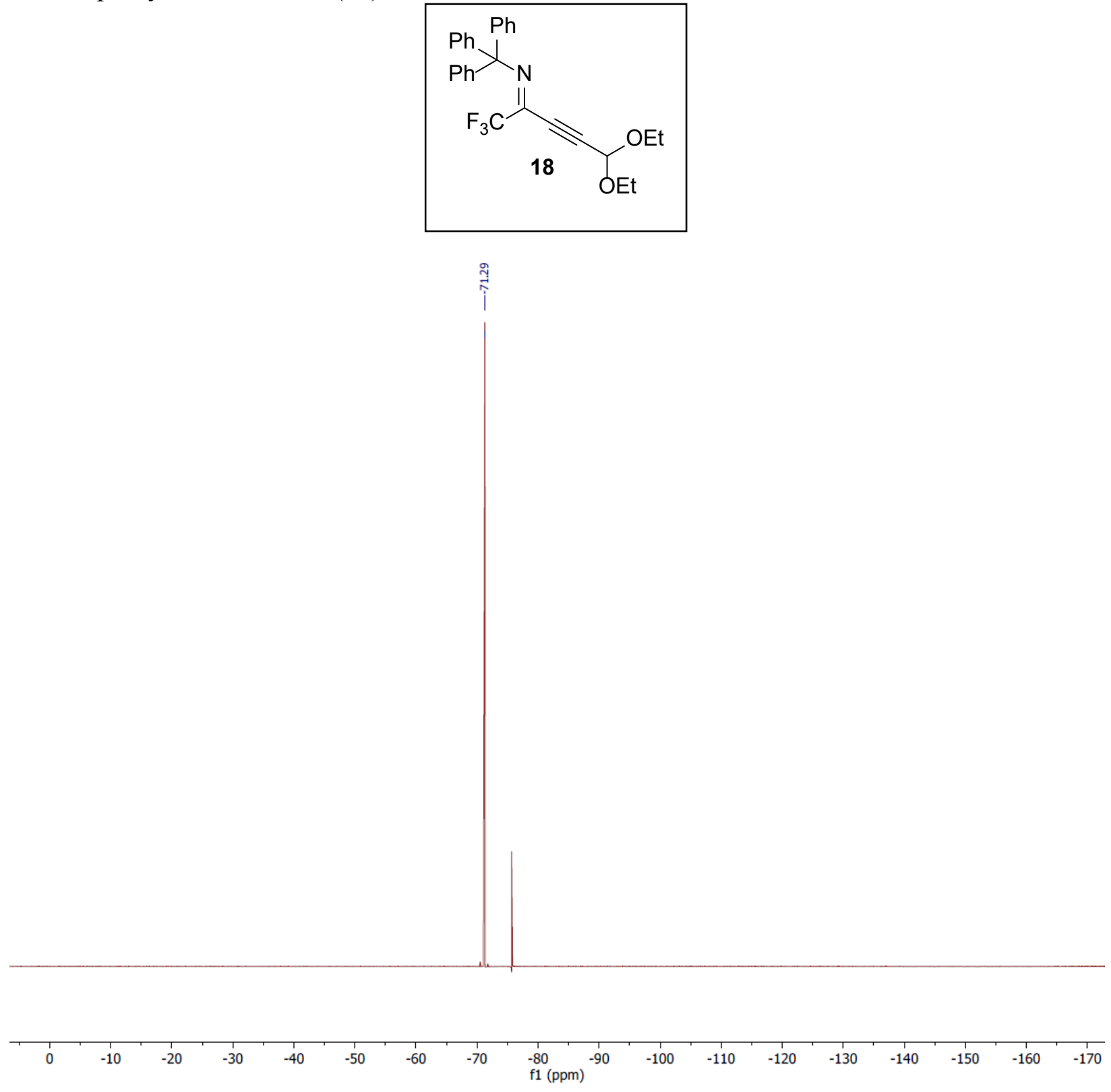
Figure S2-c. ${ }^{13} \mathrm{C}$ NMR spectra $\left(\mathrm{CDCl}_{3}\right)$ of $N$-[5,5-Diethoxy-1,1,1-trifluoropent-3-yn-2-ylidene]1,1,1-triphenylmethanamine (18).
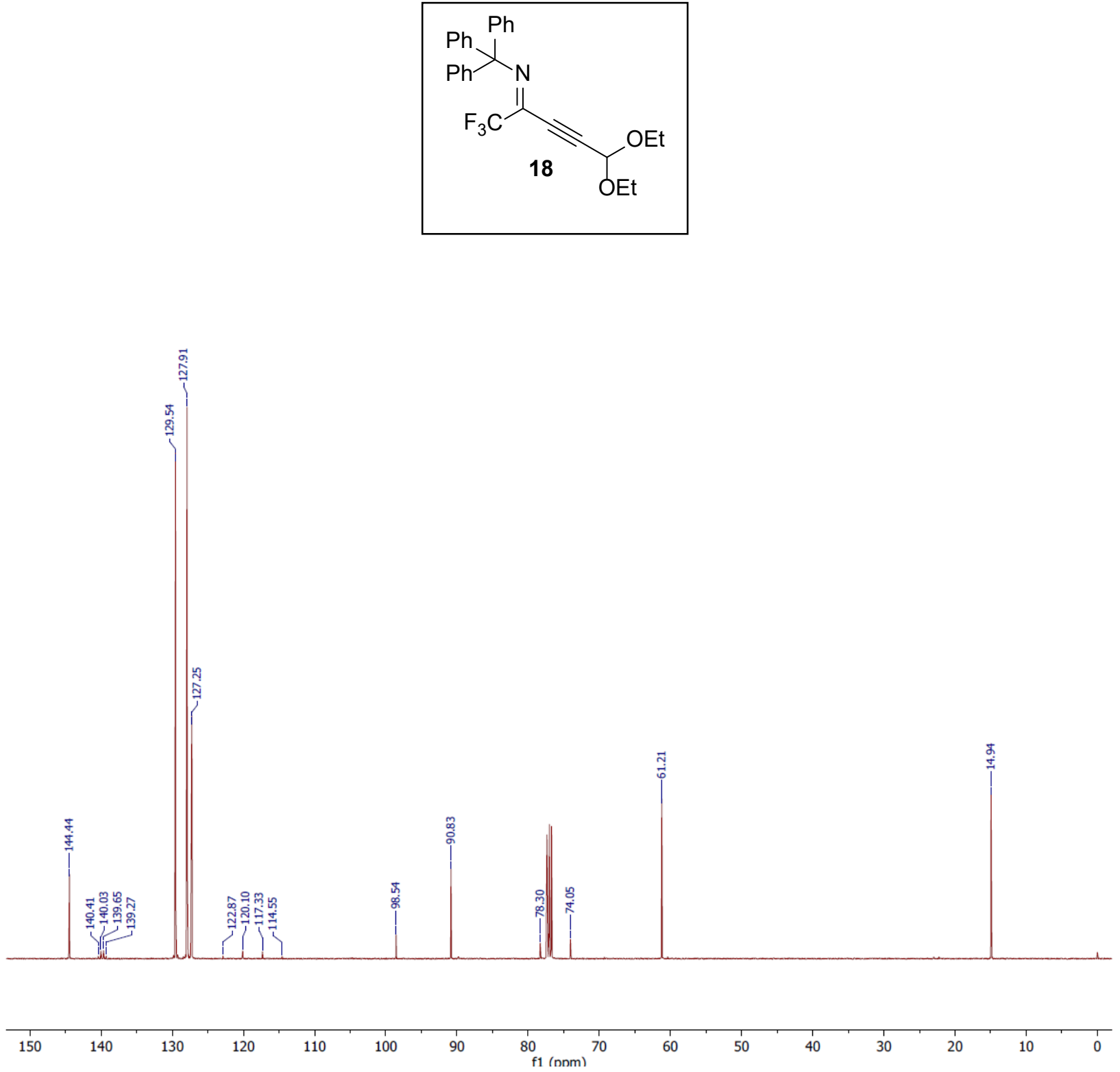
Figure S3-a. ${ }^{1} \mathrm{H}$ NMR spectra $\left(\mathrm{CDCl}_{3}\right)$ of 4-chloro-2-fluoro-3-methoxybenzaldehyde $O$-methyl oxime (20).
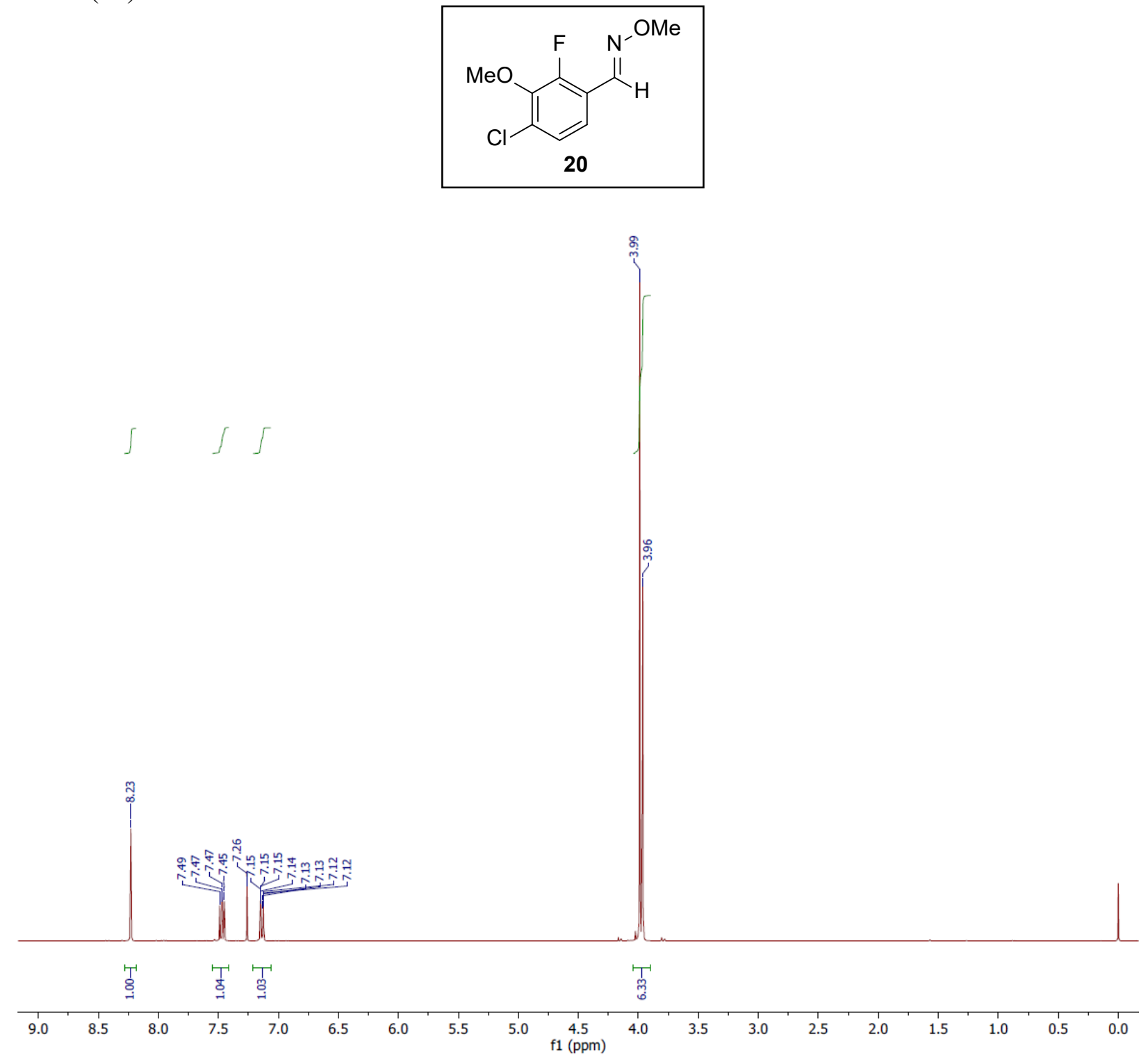
Figure S3-b. ${ }^{19} \mathrm{~F}$ NMR spectra $\left(\mathrm{CDCl}_{3}\right)$ of 4-chloro-2-fluoro-3-methoxybenzaldehyde $O$-methyl oxime (20).

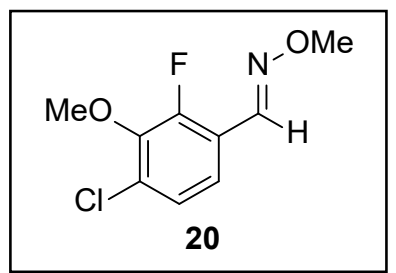

ำ

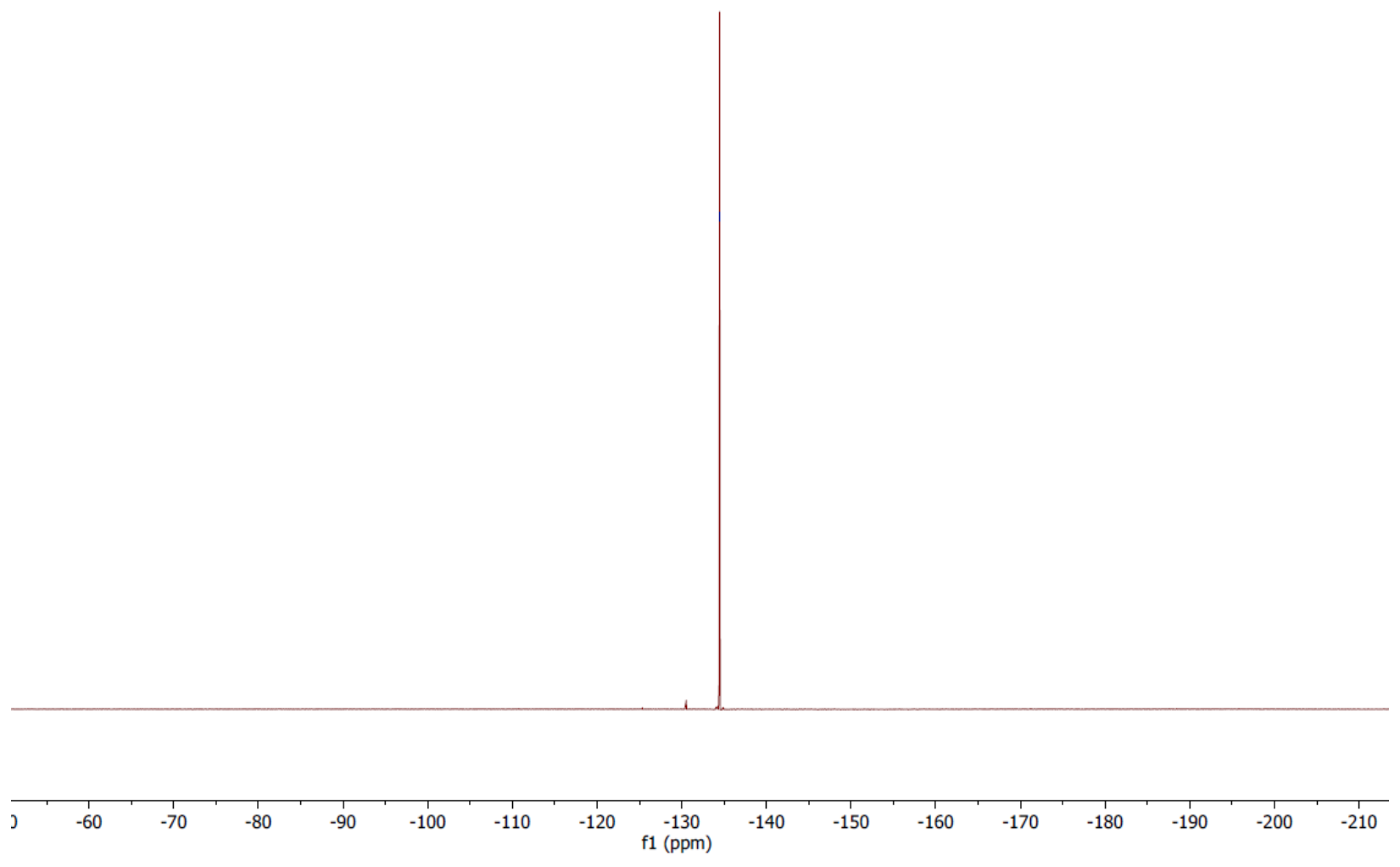


Figure $\mathrm{S} 3$-c. ${ }^{13} \mathrm{C}$ NMR spectra $\left(\mathrm{CDCl}_{3}\right)$ of 4-chloro-2-fluoro-3-methoxybenzaldehyde $O$-methyl oxime (20).
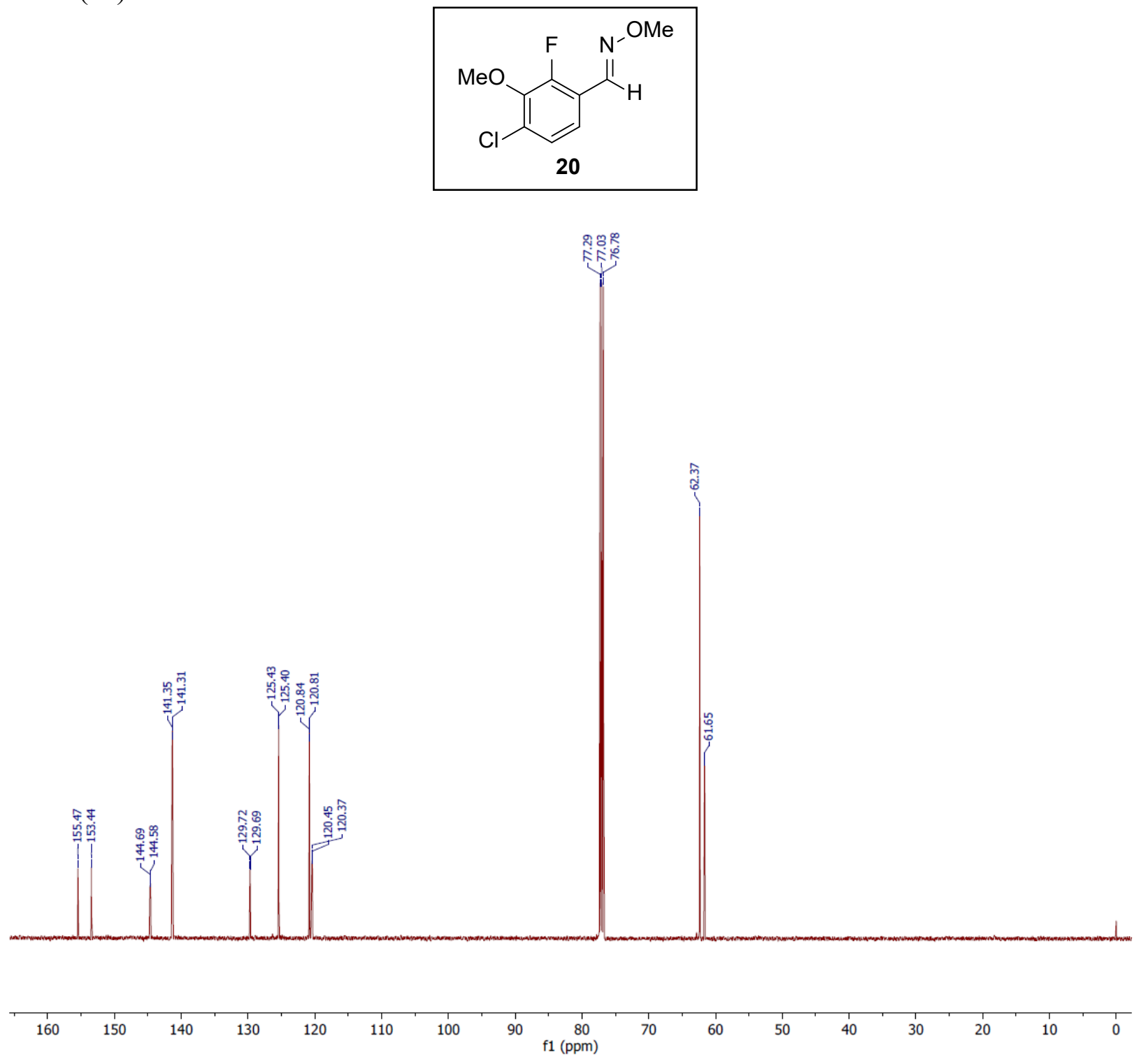
Figure S4-a. ${ }^{1} \mathrm{H}$ NMR spectra $\left(\mathrm{CDCl}_{3}\right)$ of 1-(4-chloro-2-fluoro-3-methoxyphenyl)-methanamine (13).
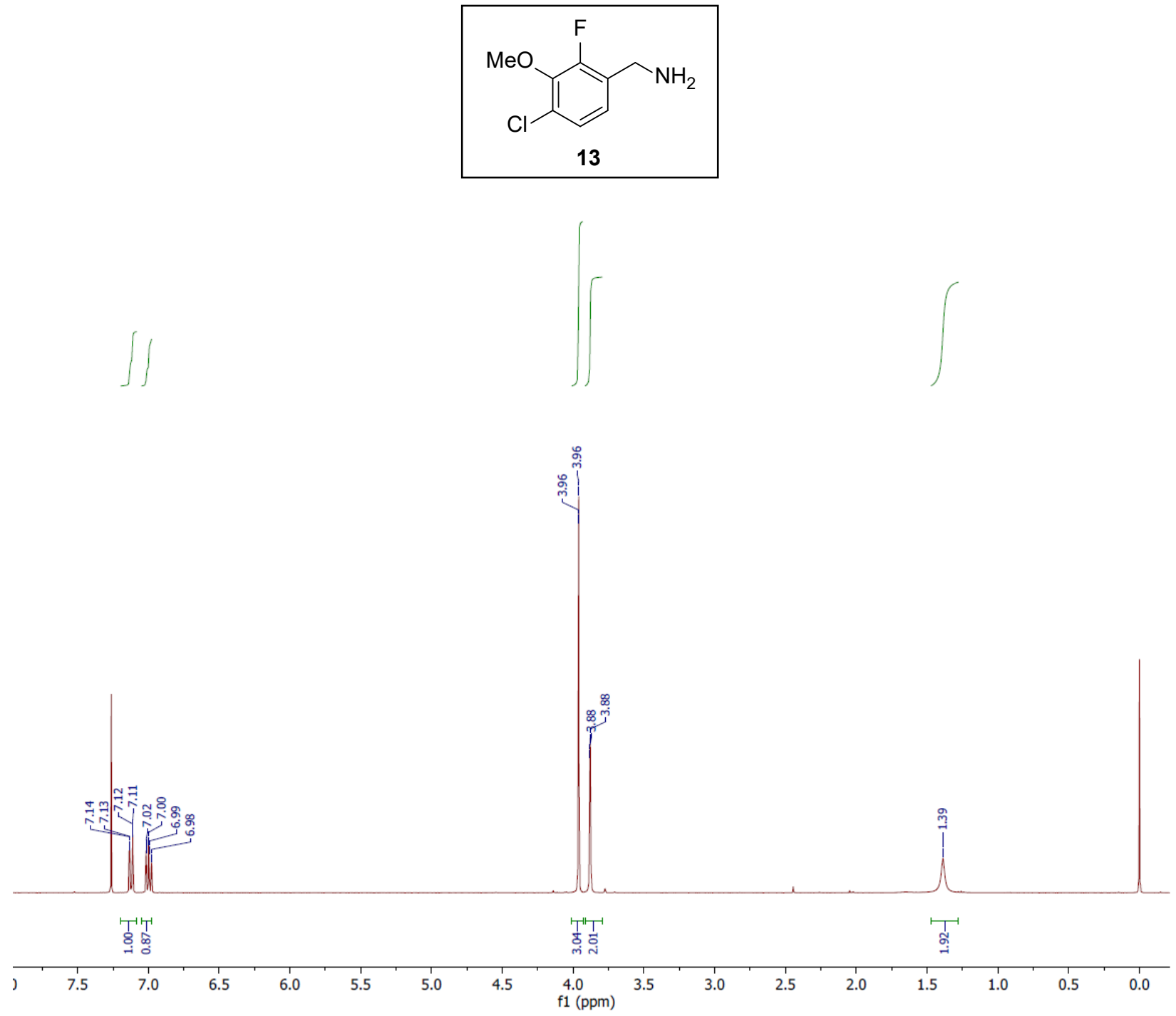
Figure S4-b. ${ }^{19} \mathrm{~F}$ NMR spectra $\left(\mathrm{CDCl}_{3}\right)$ of 1-(4-chloro-2-fluoro-3-methoxyphenyl)-methanamine (13).
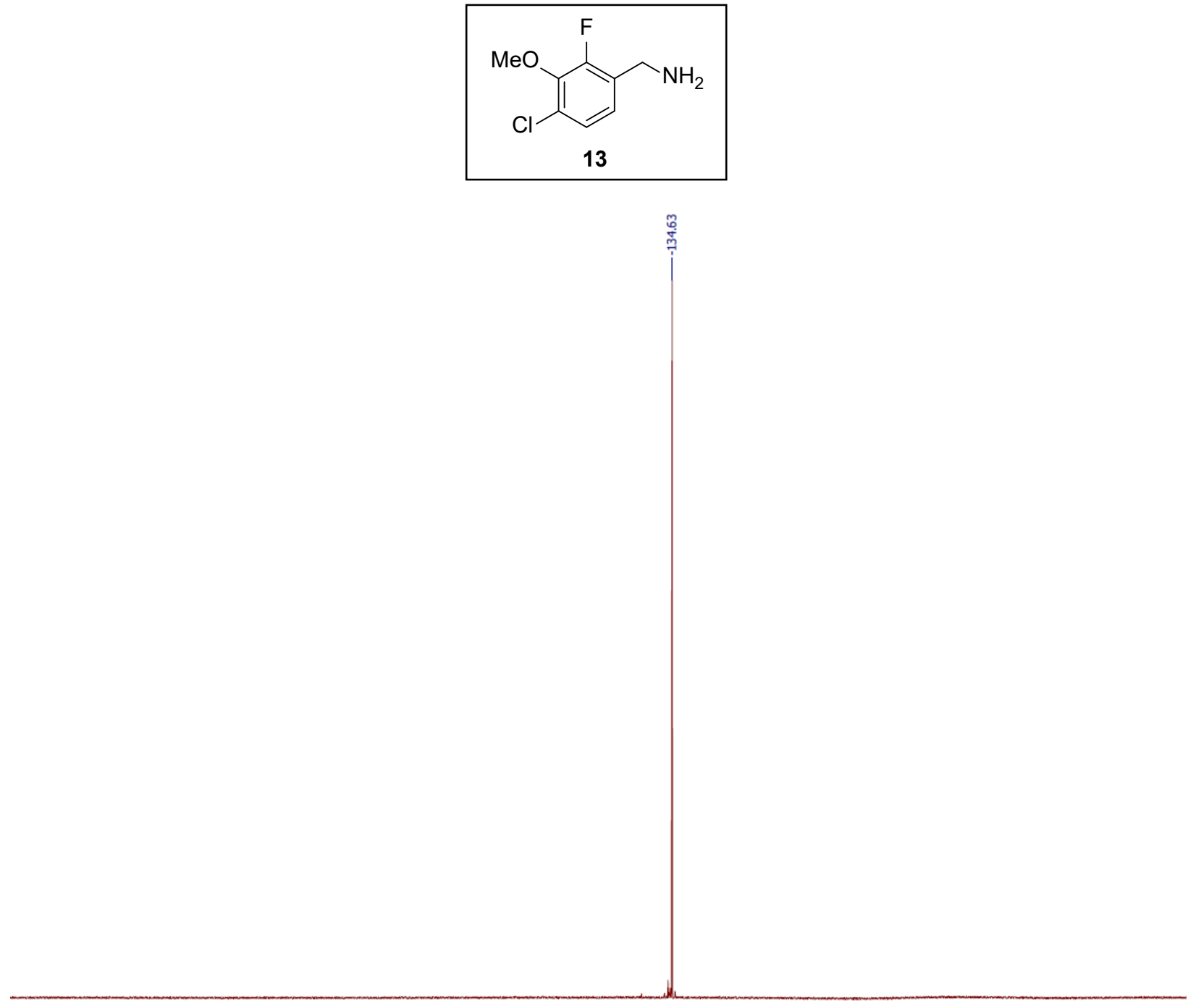

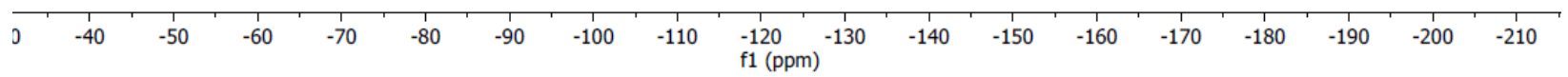


Figure S4-c. ${ }^{13} \mathrm{C}$ NMR spectra $\left(\mathrm{CDCl}_{3}\right)$ of 1-(4-chloro-2-fluoro-3-methoxyphenyl)-methanamine (13).
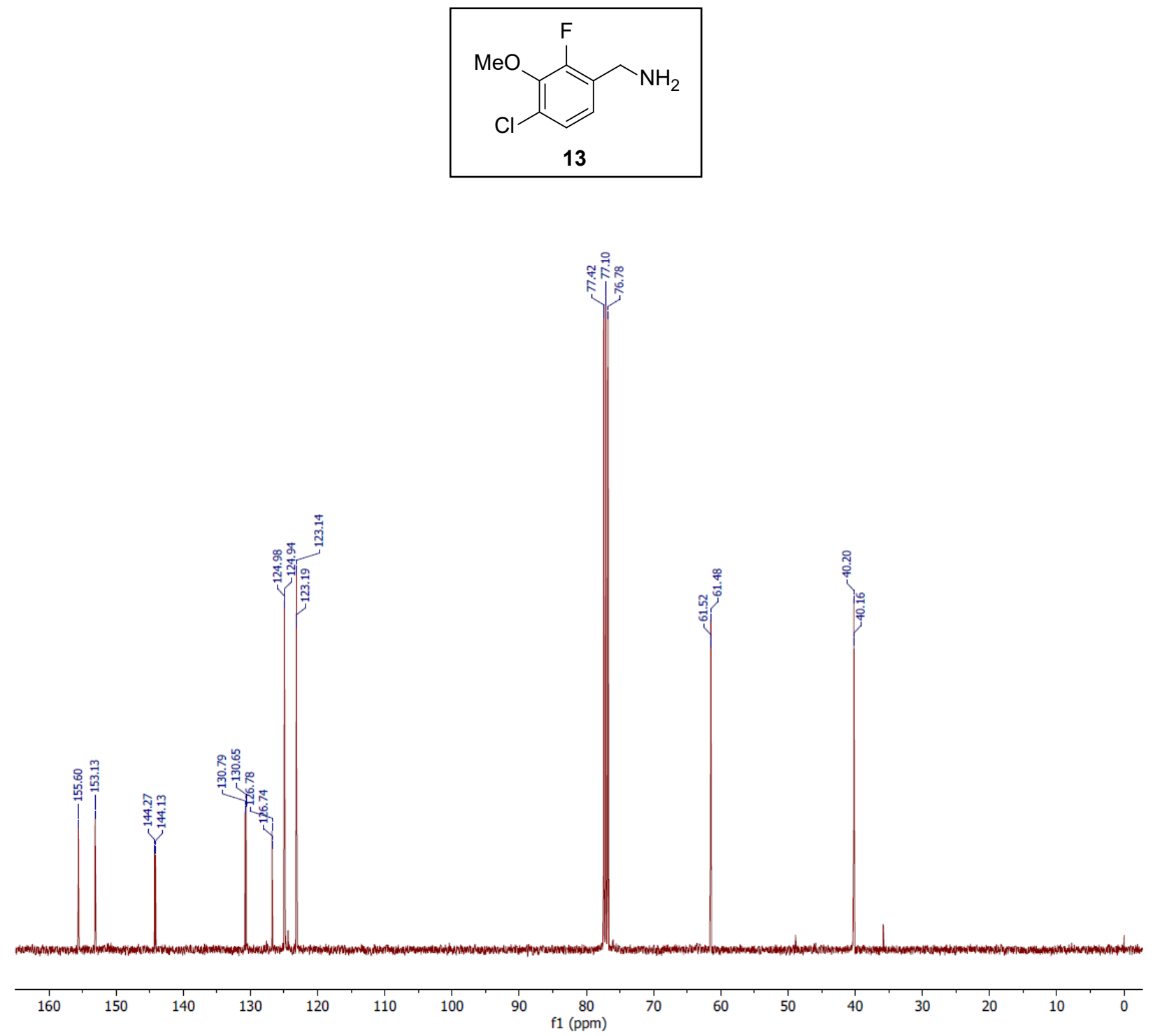
Figure S5-a. 1H NMR spectra (CDCB) of 2-(4-chloro-2-fluoro-3-methoxyphenyl)-6(diethoxymethyl)-3-fluoro-N-tritylpyridin-4-amine (21).

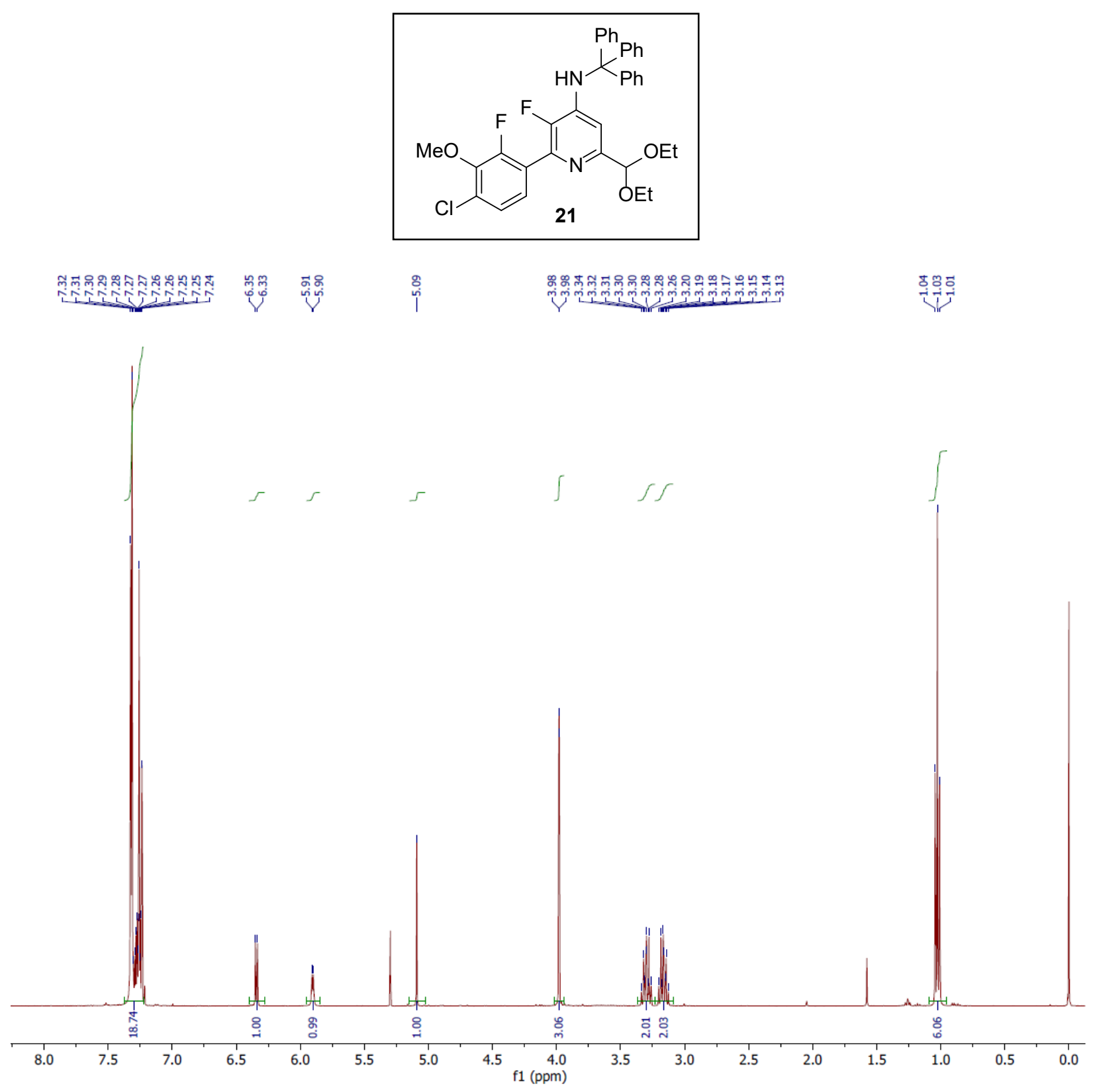


Figure S5-b. ${ }^{19} \mathrm{~F}$ NMR spectra $\left(\mathrm{CDCl}_{3}\right)$ of 2-(4-chloro-2-fluoro-3-methoxyphenyl)-6(diethoxymethyl)-3-fluoro- $N$-tritylpyridin-4-amine (21).
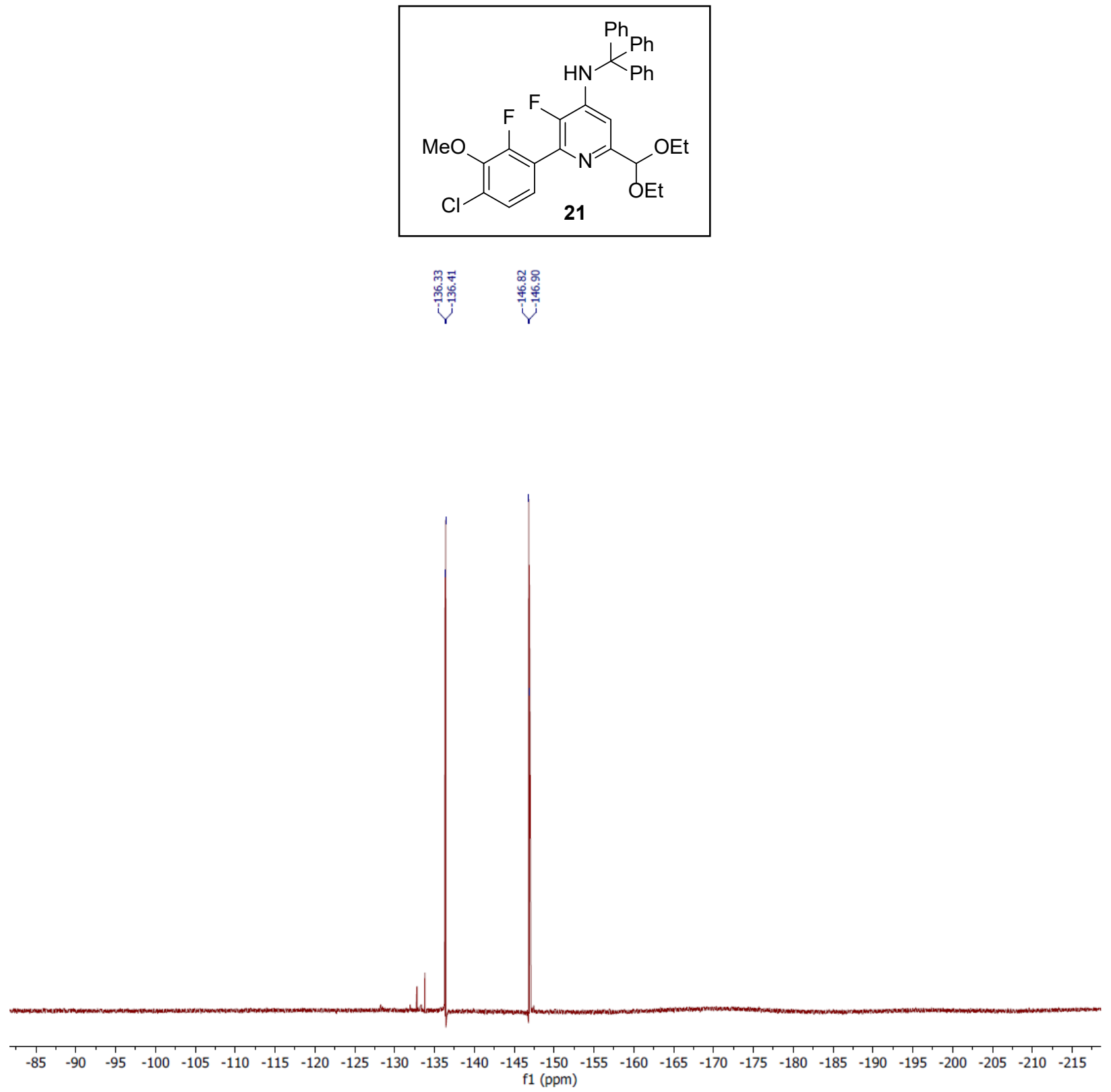
Figure S5-c. ${ }^{13} \mathrm{C}$ NMR spectra $\left(\mathrm{CDCl}_{3}\right)$ of 2-(4-chloro-2-fluoro-3-methoxyphenyl)-6(diethoxymethyl)-3-fluoro- $N$-tritylpyridin-4-amine (21).

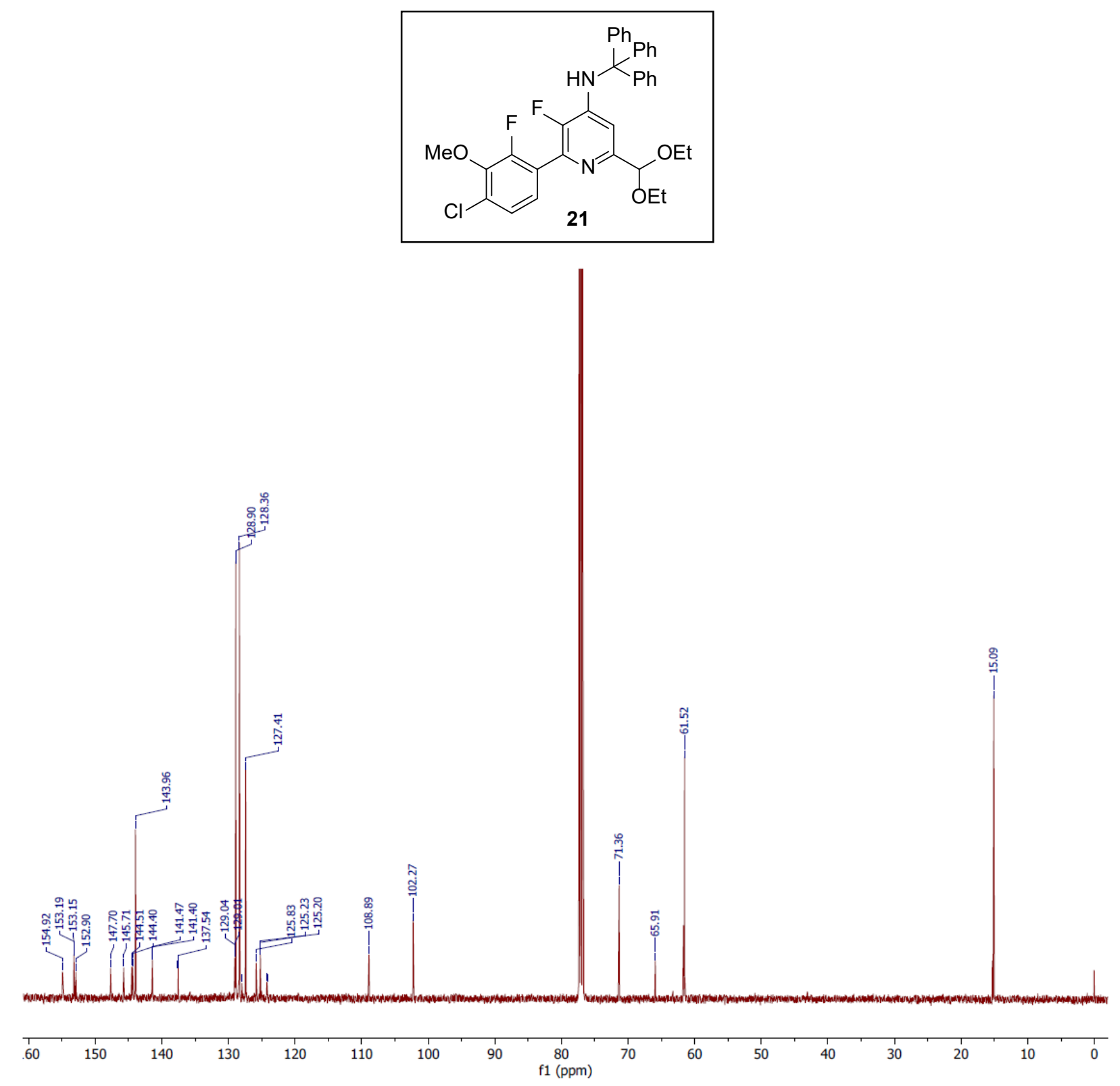


Figure S6-a. ${ }^{1} \mathrm{H}$ NMR spectra (DMSO- $d_{6}$ ) of 4-amino-6-(4-chloro-2-fluoro-3-methoxyphenyl)-5fluoropyridine-2-carbaldehyde (22).

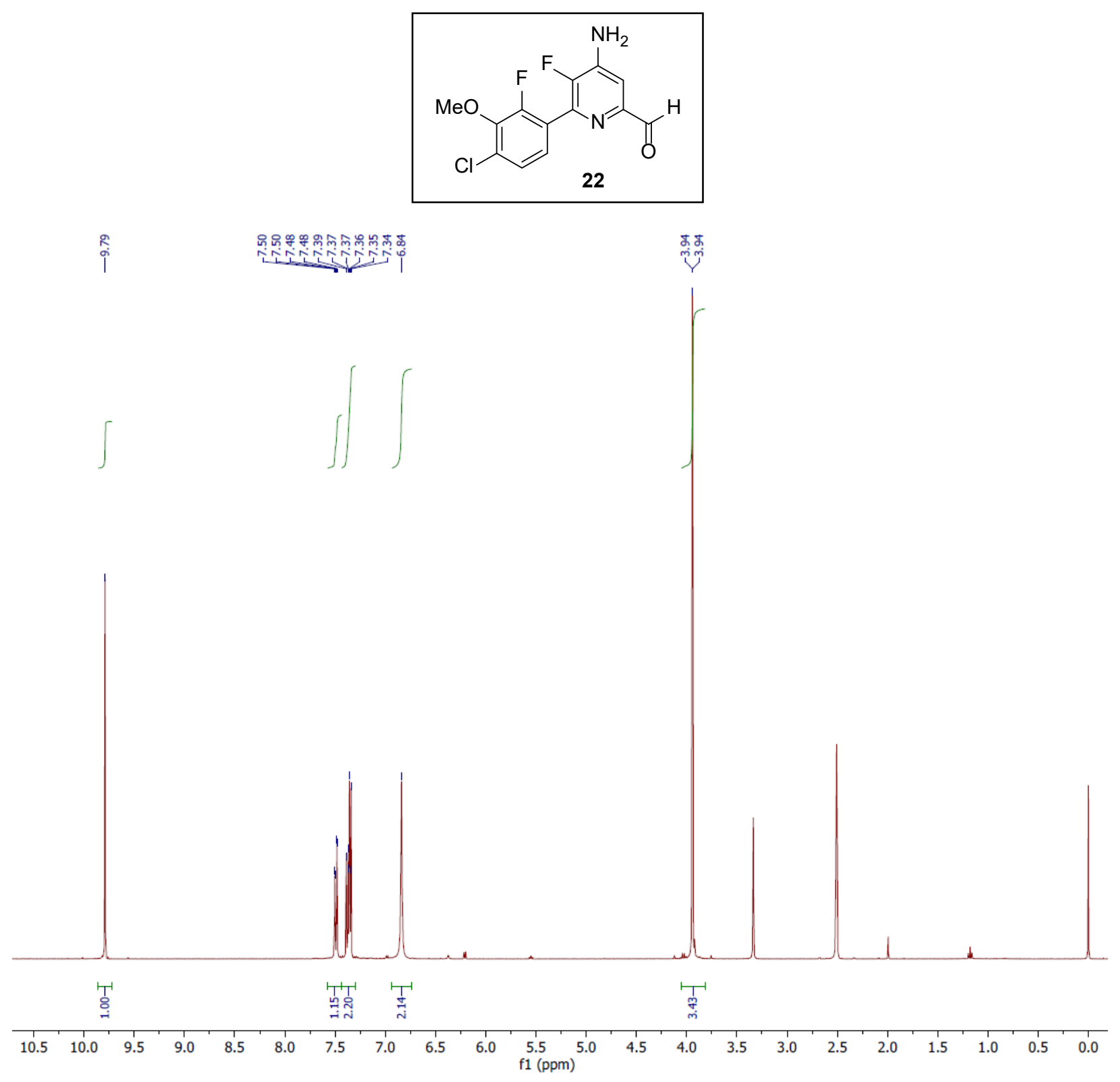


Figure S6-b. ${ }^{19} \mathrm{~F}$ NMR spectra (DMSO- $d_{6}$ ) of 4-amino-6-(4-chloro-2-fluoro-3-methoxyphenyl)5-fluoropyridine-2-carbaldehyde (22).
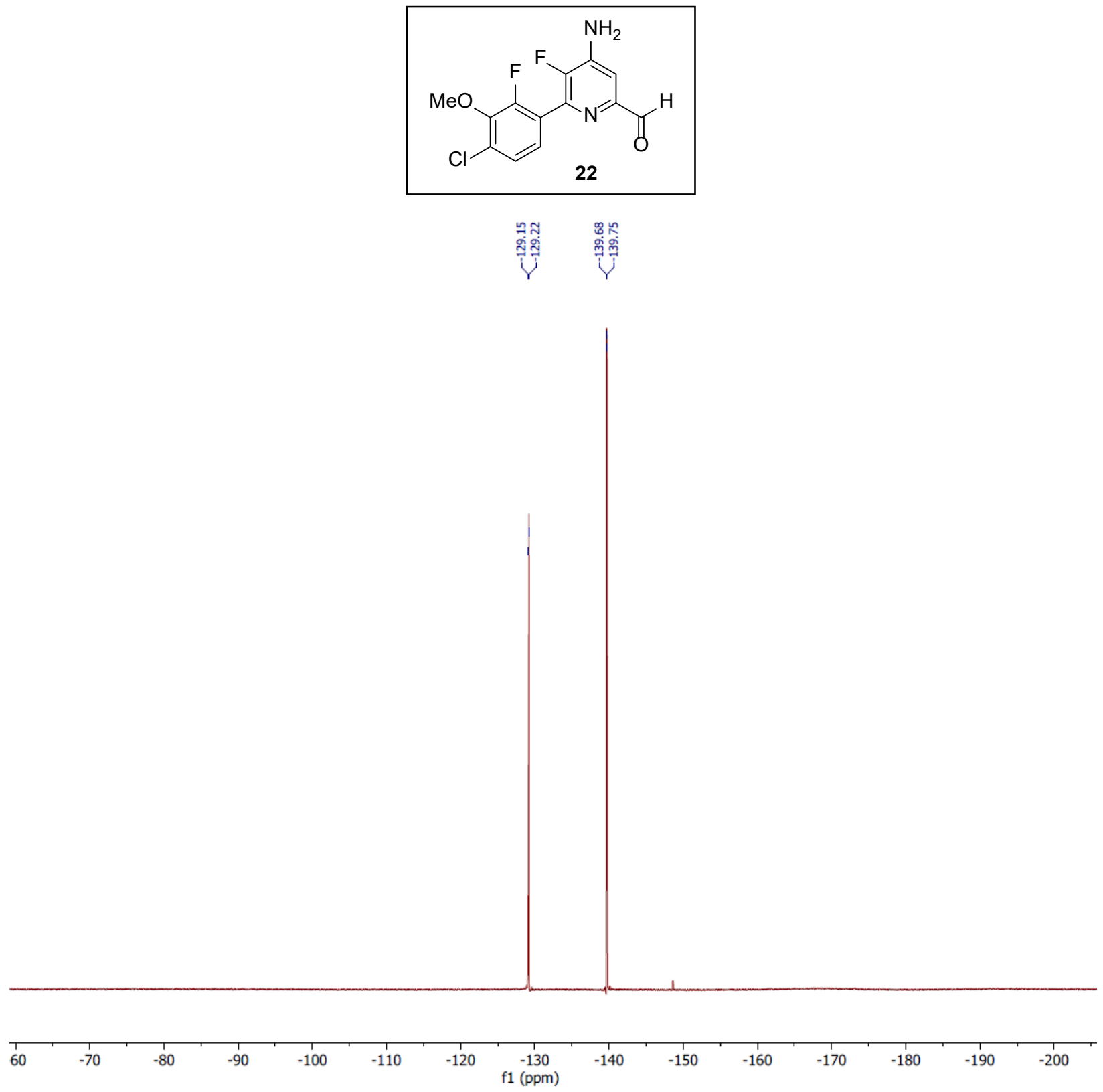
Figure S6-c. ${ }^{13} \mathrm{C}$ NMR spectra (DMSO- $d_{6}$ ) of 4-amino-6-(4-chloro-2-fluoro-3-methoxyphenyl)5-fluoropyridine-2-carbaldehyde (22).
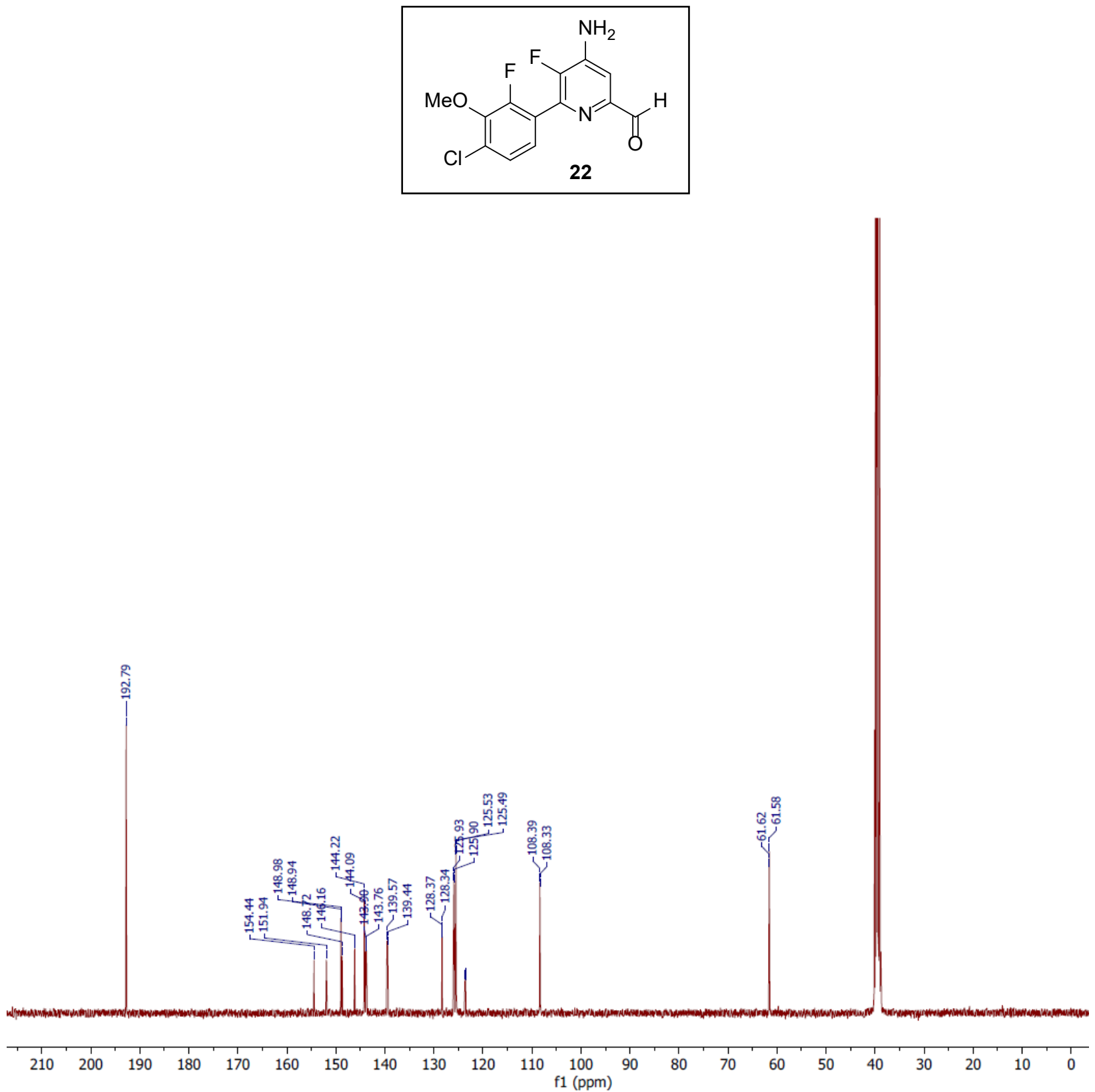
Figure S7-a. 1H NMR spectra (DMSO- $d_{6}$ ) of 4-Amino-3-chloro-6-(4-chloro-2-fluoro-3methoxyphenyl)-5-fluoropyridine-2-carbaldehyde (23).

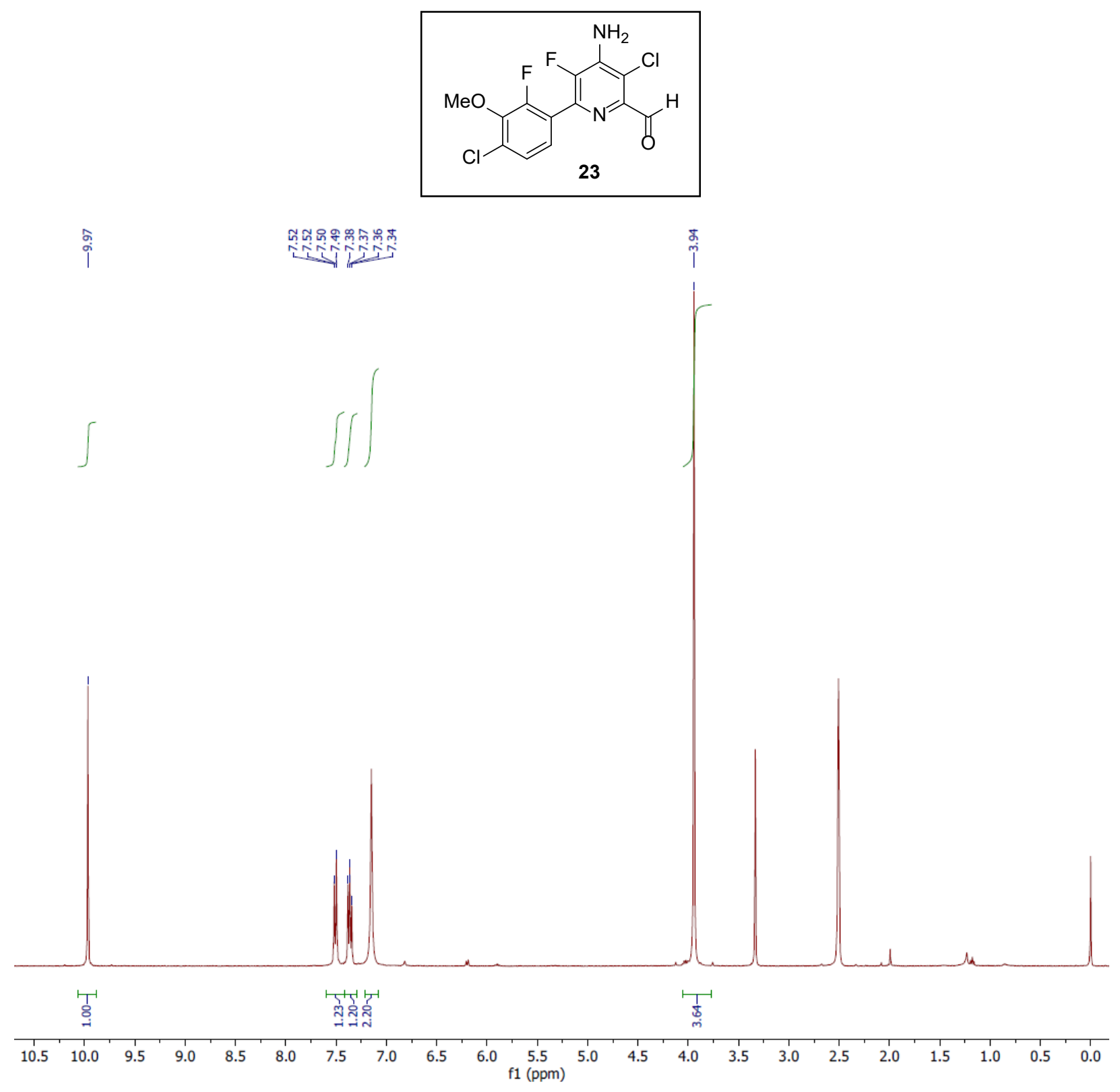


Figure S7-b. ${ }^{19}$ F NMR spectra (DMSO- $d_{6}$ ) of 4-Amino-3-chloro-6-(4-chloro-2-fluoro-3methoxyphenyl)-5-fluoropyridine-2-carbaldehyde (23).
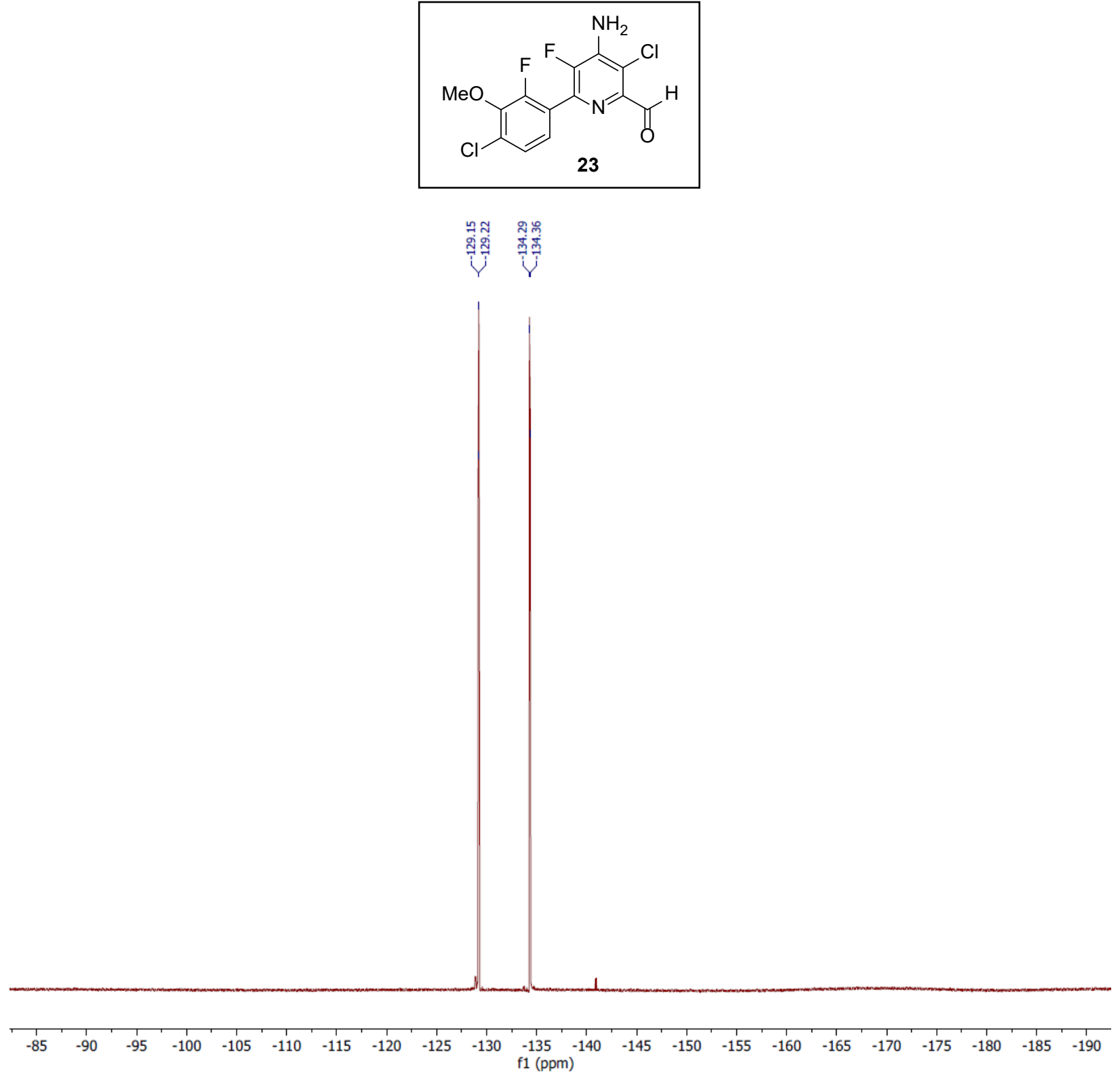
Figure S7-c. ${ }^{13} \mathrm{C}$ NMR spectra (DMSO- $d_{6}$ ) of 4-Amino-3-chloro-6-(4-chloro-2-fluoro-3methoxyphenyl)-5-fluoropyridine-2-carbaldehyde (23).
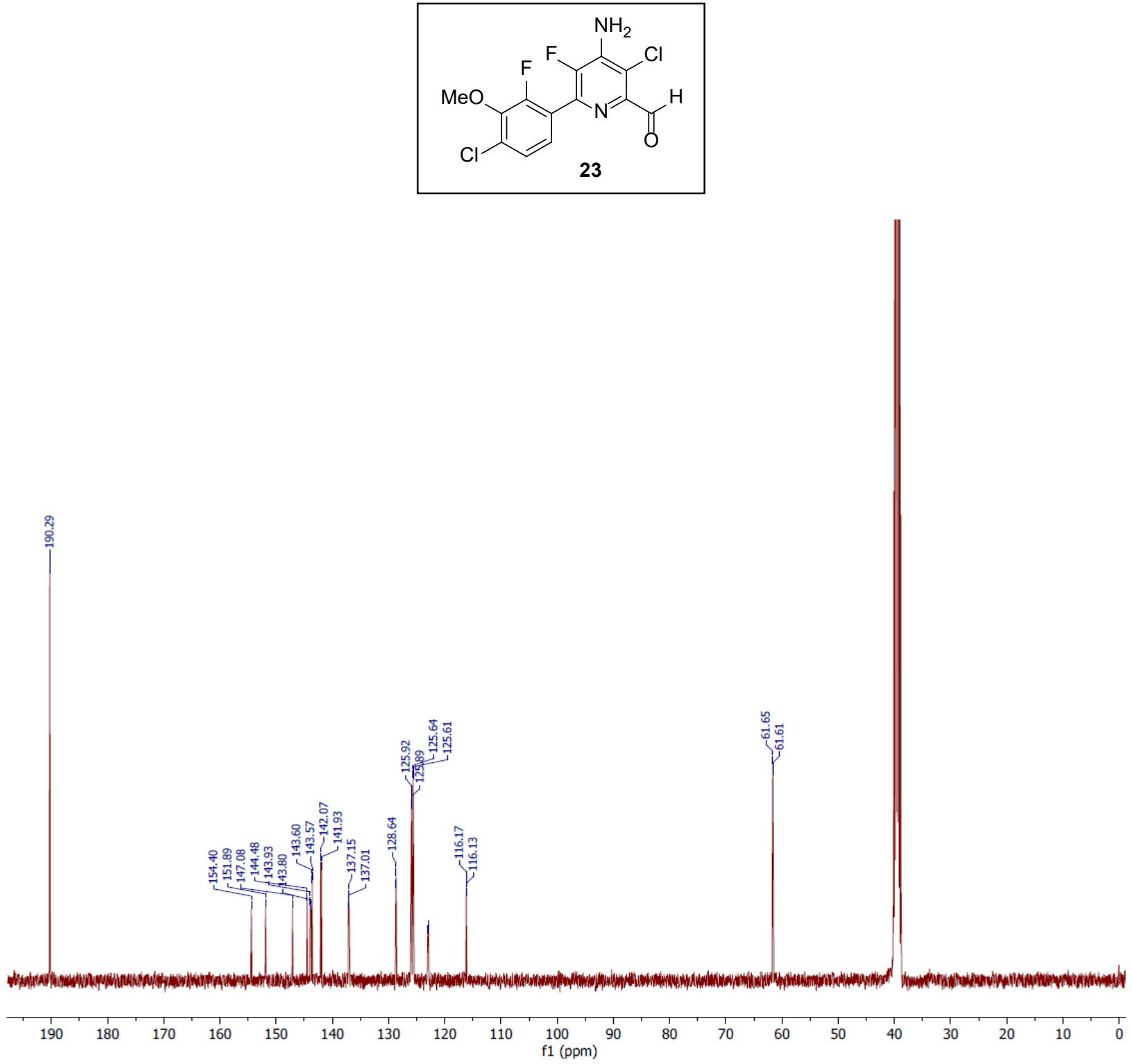
Figure S8-a. ${ }^{1} \mathrm{H}$ NMR spectra (DMSO- $d_{6}$ ) of 4-amino-3-chloro-6-(4-chloro-2-fluoro-3methoxyphenyl)-5-fluoropyridine-2-carboxylic acid (24).

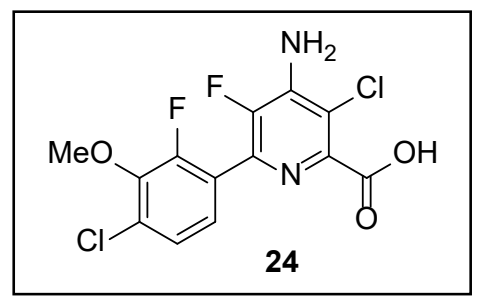

i̊n
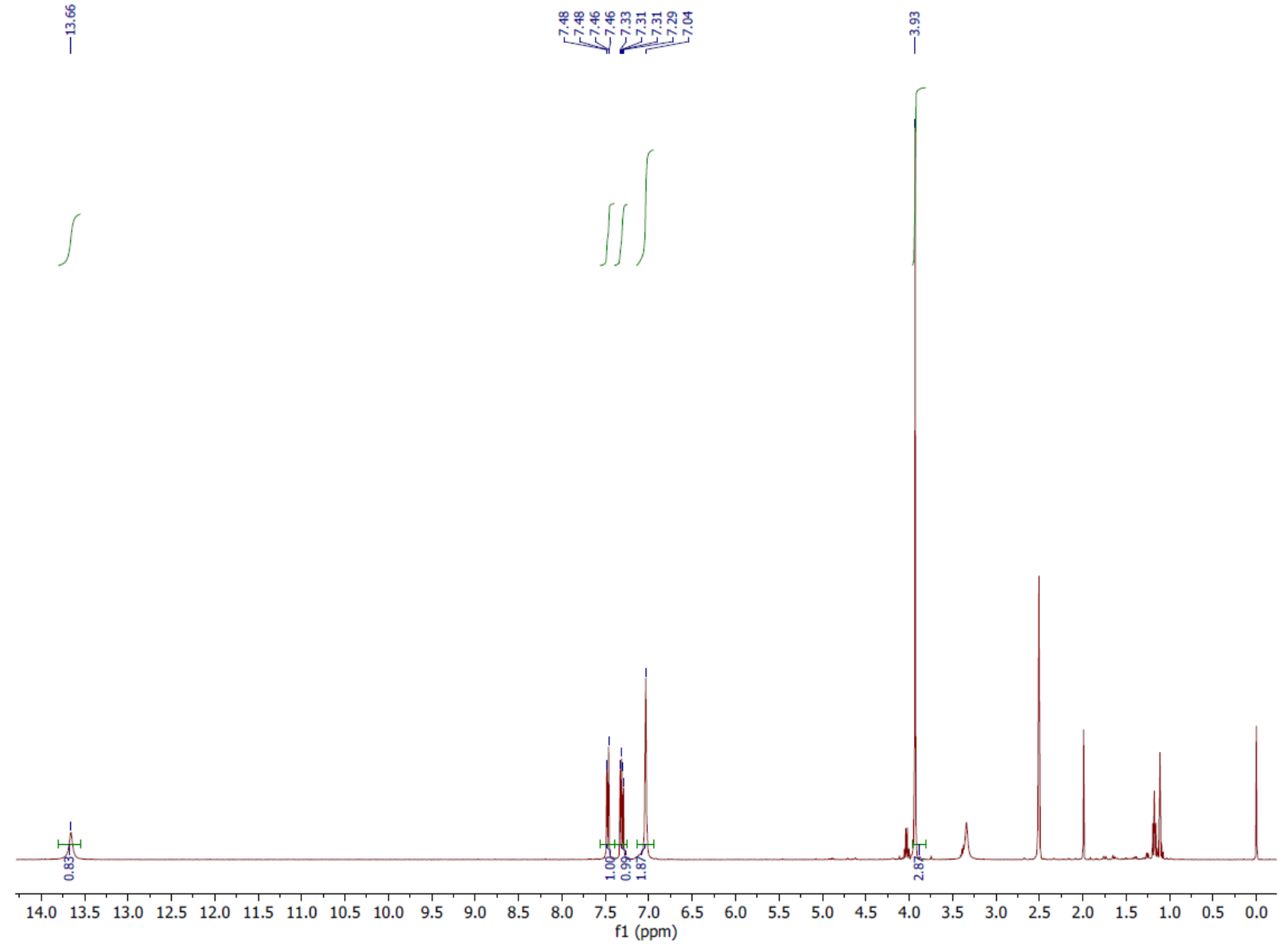
Figure S8-b. ${ }^{19} \mathrm{~F}$ NMR spectra (DMSO- $d_{6}$ ) of 4-amino-3-chloro-6-(4-chloro-2-fluoro-3methoxyphenyl)-5-fluoropyridine-2-carboxylic acid (24).

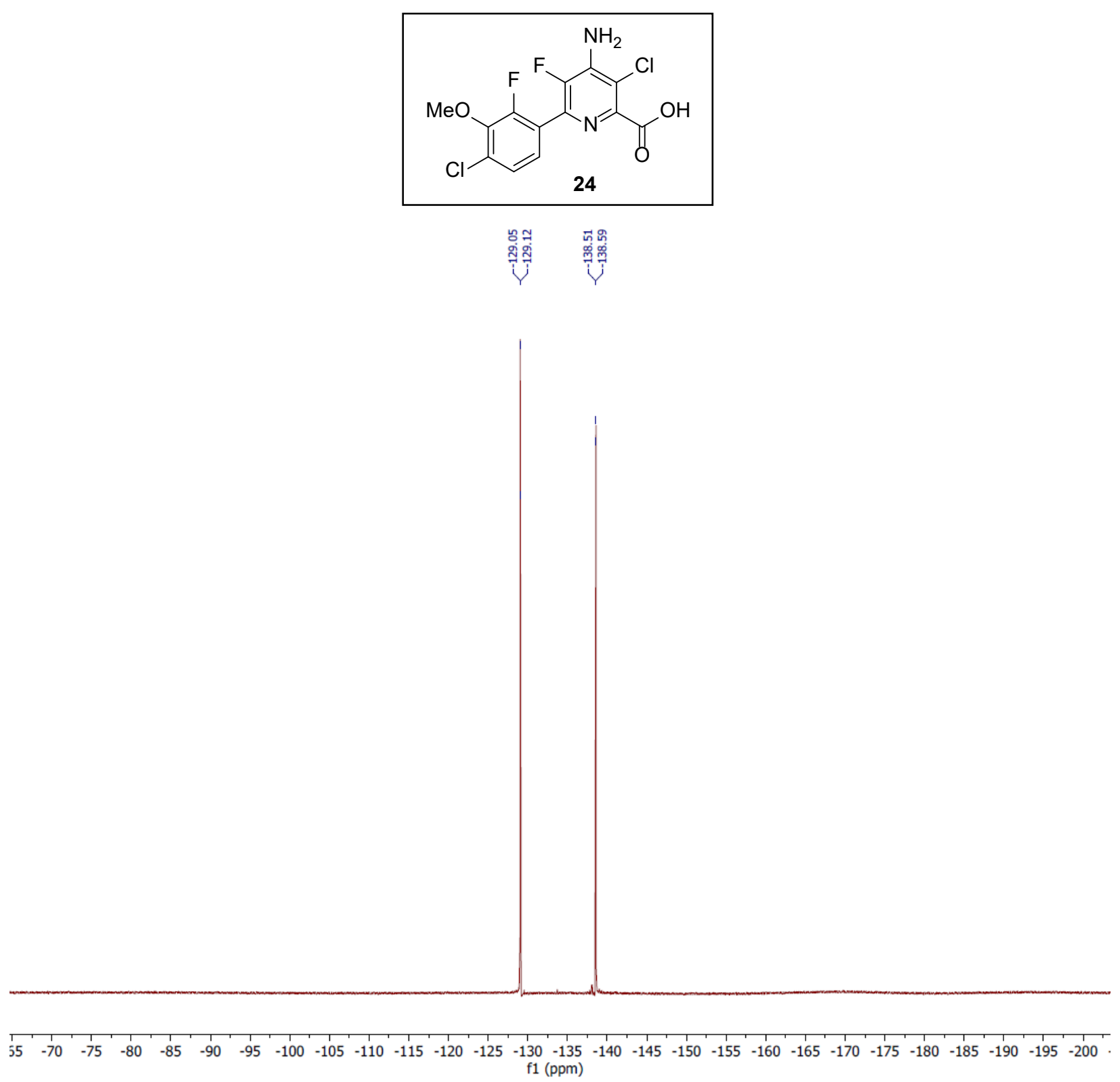


Figure S8-c. ${ }^{13} \mathrm{C}$ NMR spectra (DMSO- $d_{6}$ ) of 4-amino-3-chloro-6-(4-chloro-2-fluoro-3methoxyphenyl)-5-fluoropyridine-2-carboxylic acid (24).

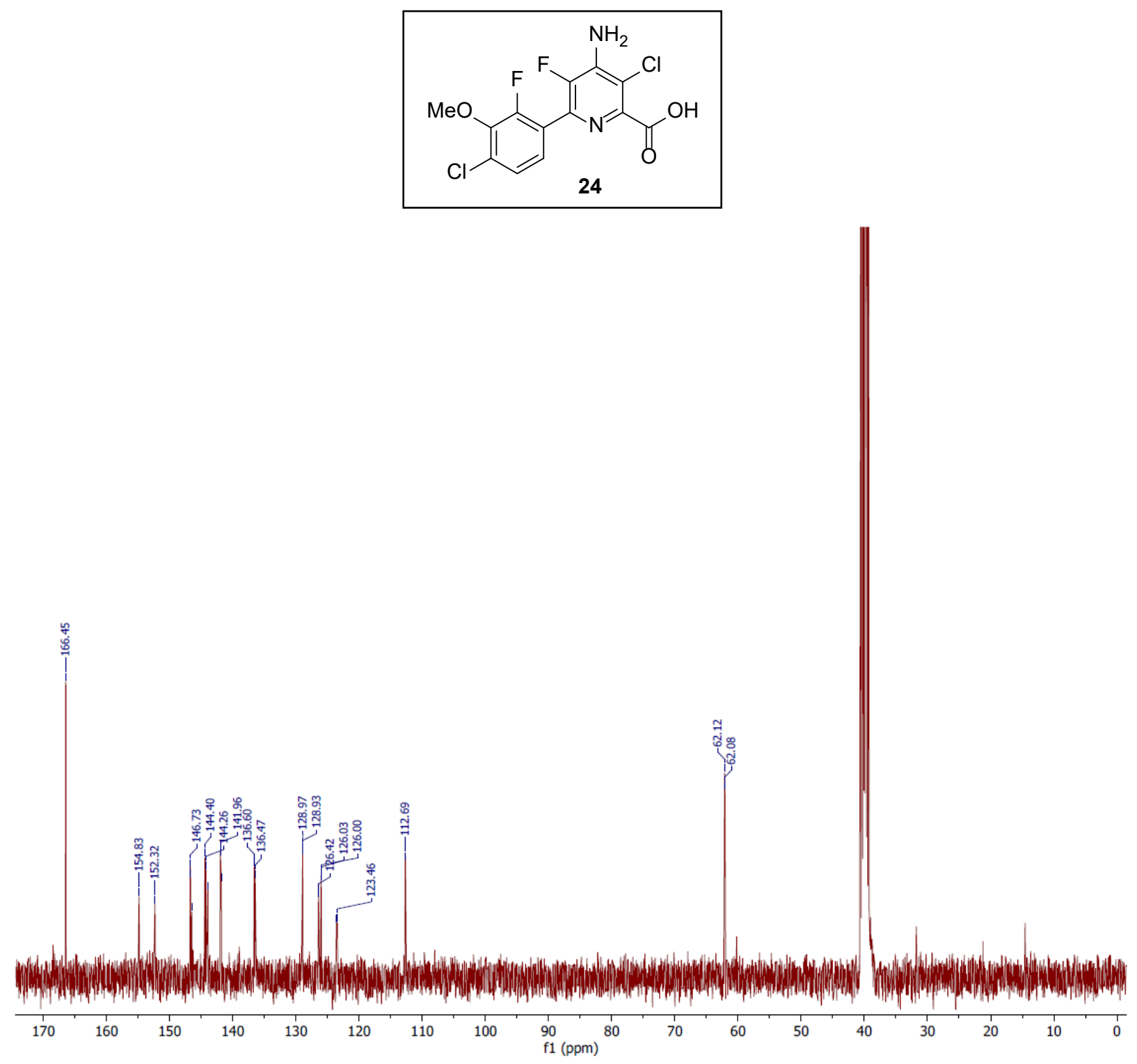


Figure S9-a. ${ }^{1} \mathrm{H}$ NMR spectra $\left(\mathrm{CDCl}_{3}\right)$ of benzyl 4-amino-3-chloro-6-(4-chloro-2-fluoro-3methoxyphenyl)-5-fluoropyridine-2-carboxylate (Rinskor, 1).

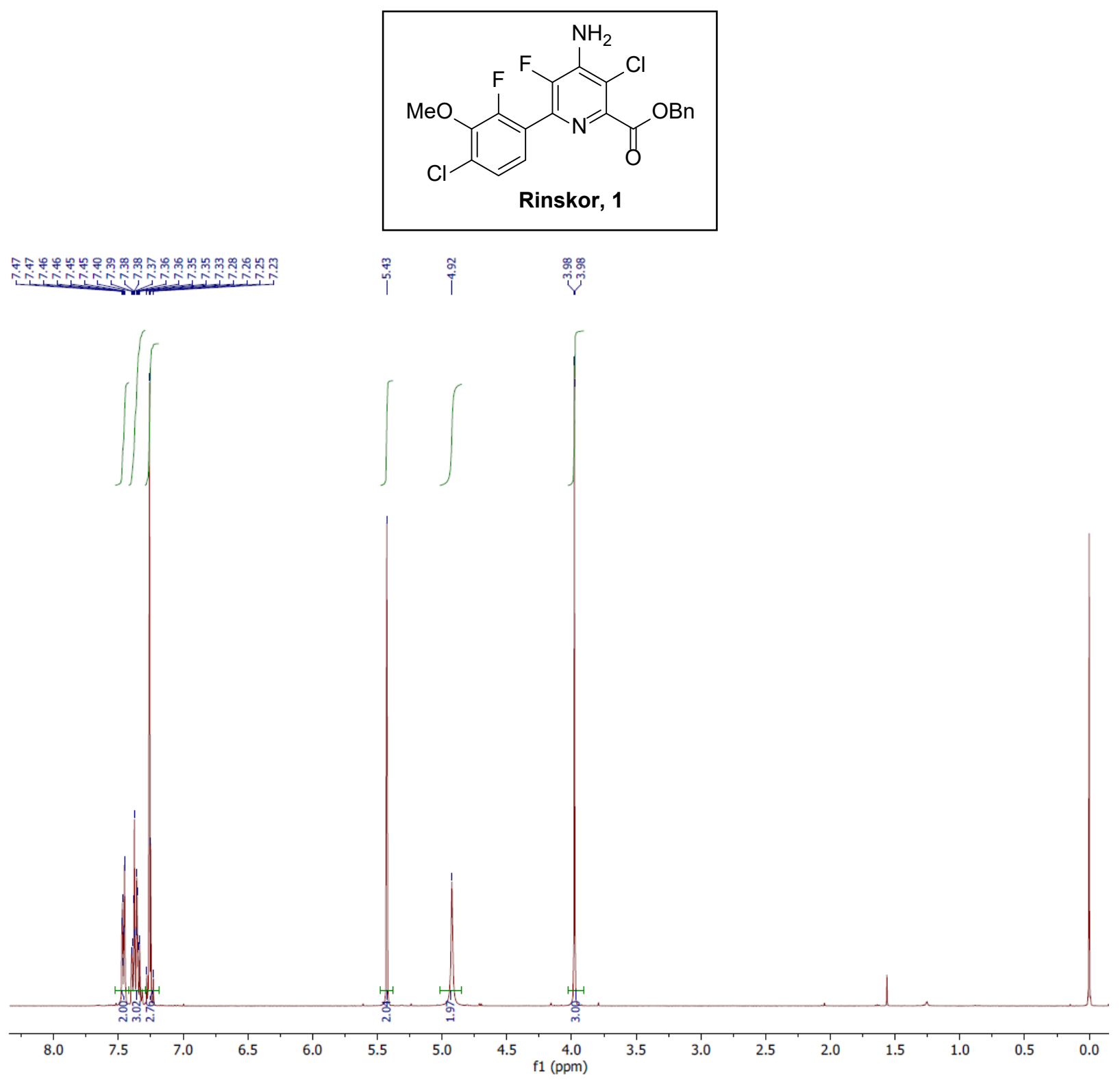


Figure S9-b. ${ }^{19} \mathrm{~F}$ NMR spectra $\left(\mathrm{CDCl}_{3}\right)$ of benzyl 4-amino-3-chloro-6-(4-chloro-2-fluo ro-3methoxyphenyl)-5-fluoropyridine-2-carboxylate (Rinskor, 1).

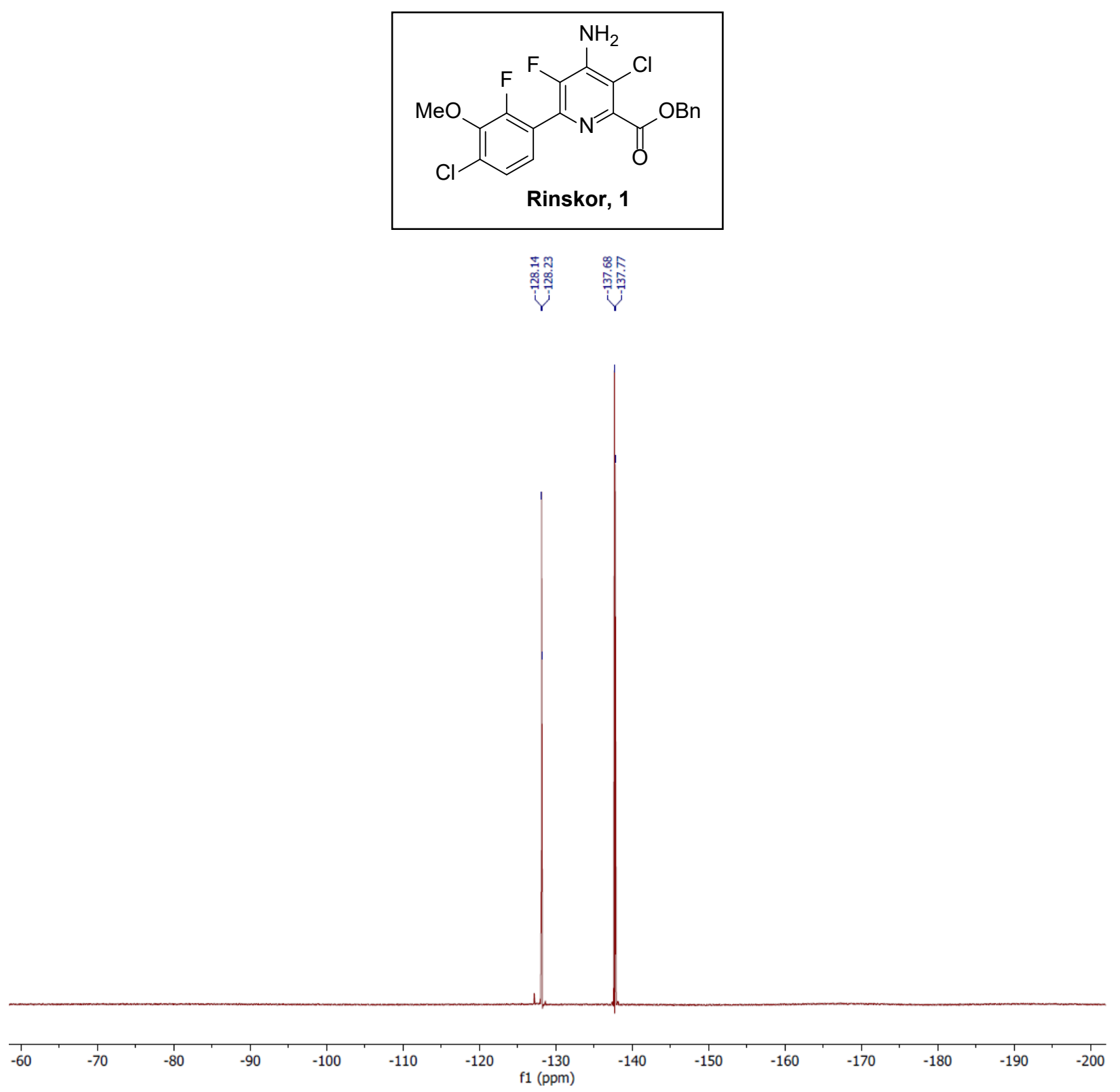


Figure S9-c. ${ }^{13} \mathrm{C}$ NMR spectra $\left(\mathrm{CDCl}_{3}\right)$ of benzyl 4-amino-3-chloro-6-(4-chloro-2-fluoro-3methoxyphenyl)-5-fluoropyridine-2-carboxylate (Rinskor, 1).

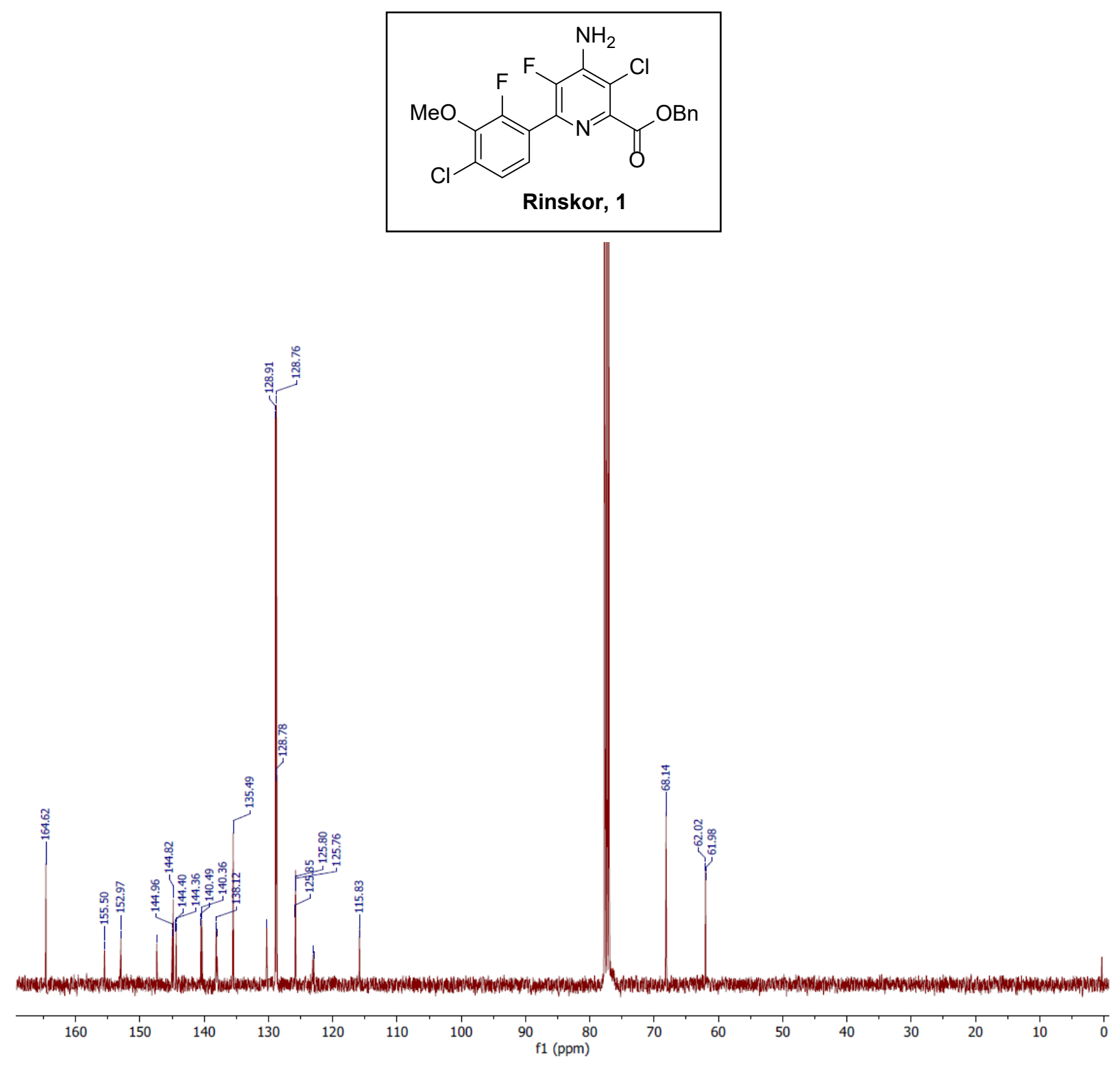


Figure S10. HPLC chromatograms for 2-(4-chloro-2-fluoro-3-methoxyphenyl)-6(diethoxymethyl)-3-fluoro- $N$-trityl $\left(4-{ }^{14} \mathrm{C}\right)$ pyridin-4-amine (26).
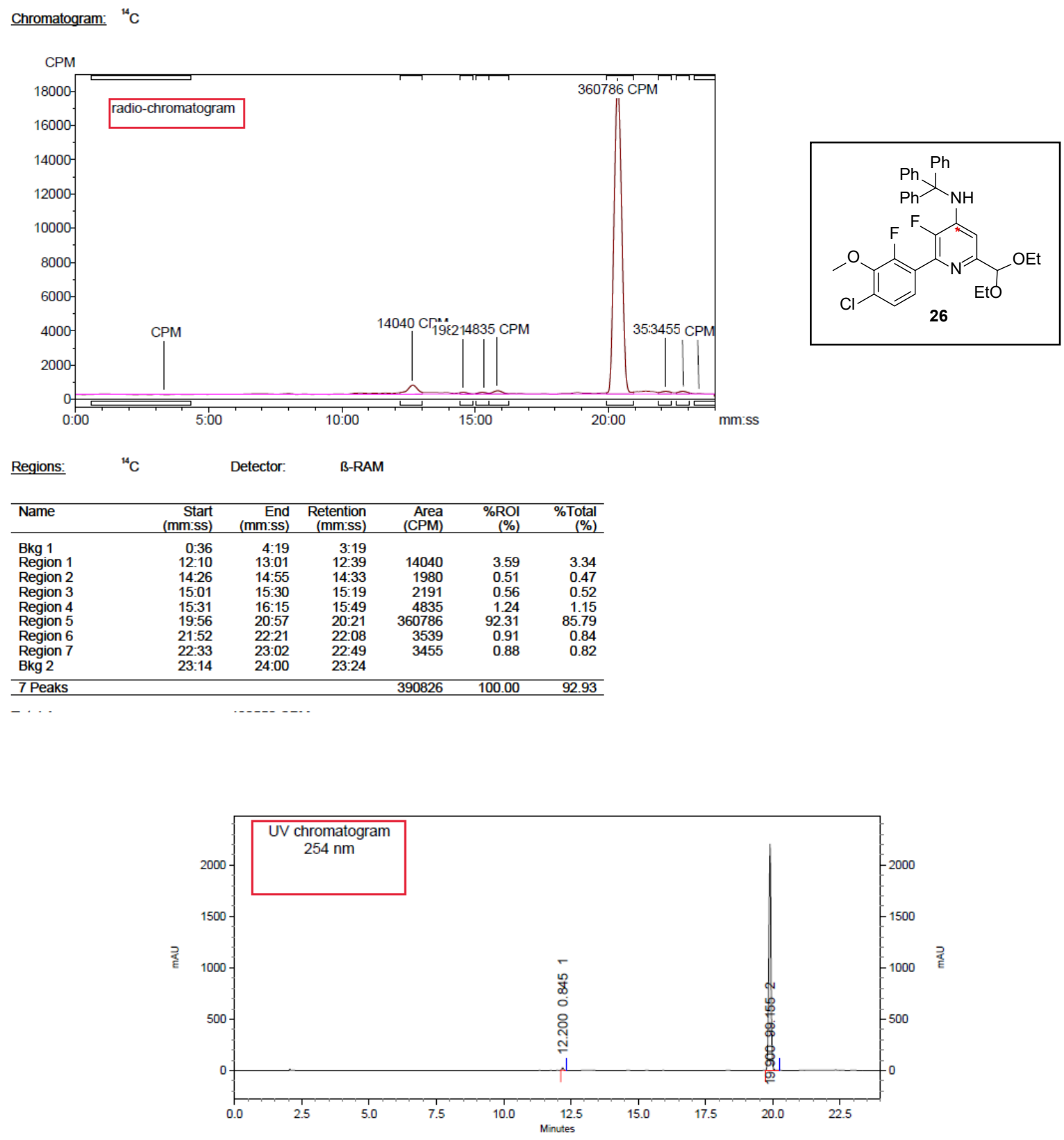

\begin{tabular}{lccc}
$\begin{array}{l}\text { 1: } 254 \mathrm{~nm}, 4 \mathrm{~nm} \\
\text { Results } \\
\mathrm{Pk} \#\end{array}$ & Retention Time & Area & Area Percent \\
\hline 1 & 12.200 & 103122 & 0.85 \\
2 & 19.900 & 12095071 & 99.15 \\
\hline Totals & & & \\
& & 12198193 & 100.00 \\
\hline
\end{tabular}


Figure S11. HPLC chromatograms for 4-Amino-6-(4-chloro-2-fluoro-3-methoxyphenyl)-5fluoro(4- $\left.{ }^{14} \mathrm{C}\right)$ pyridine-2-carbaldehyde (27).
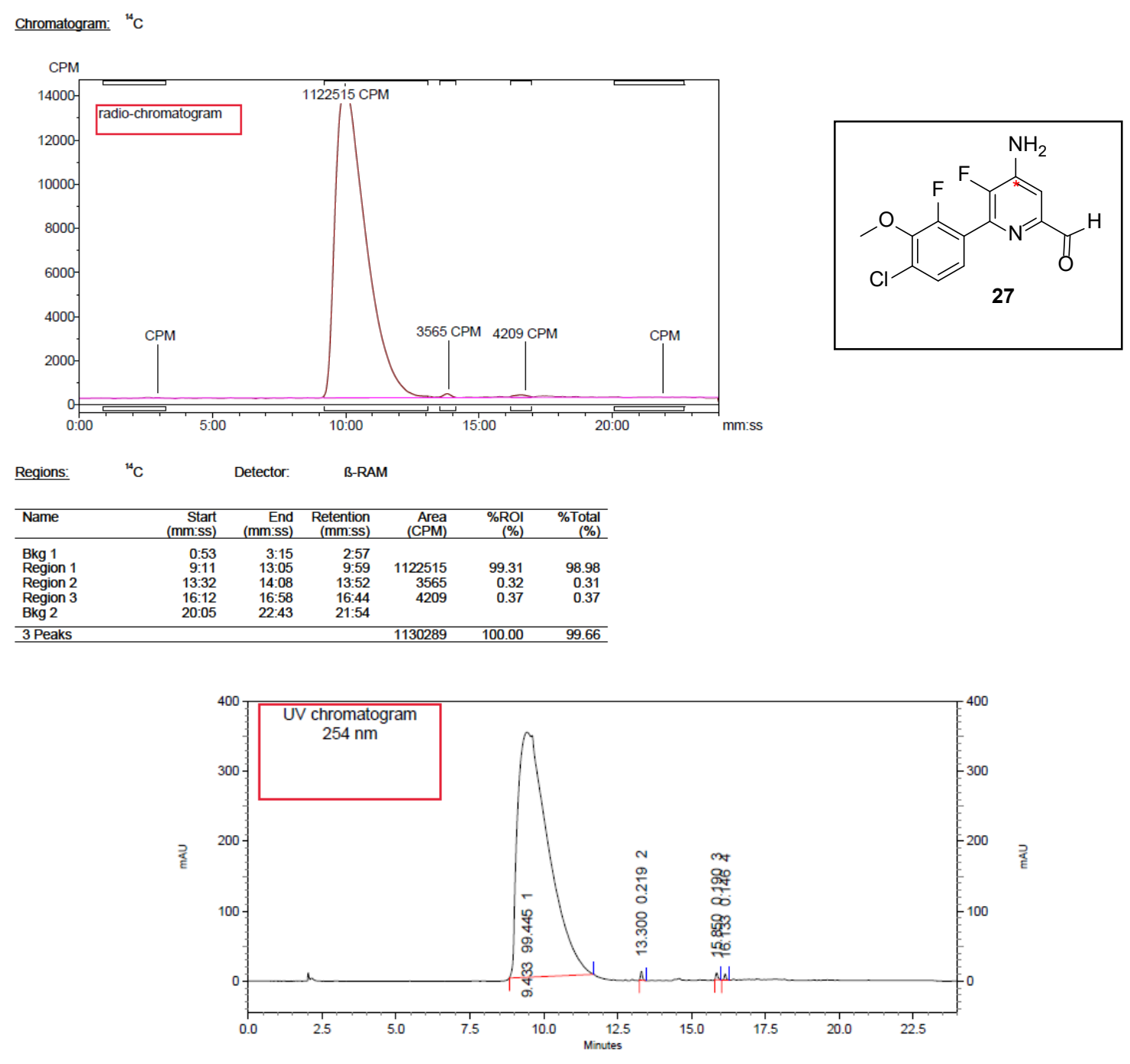

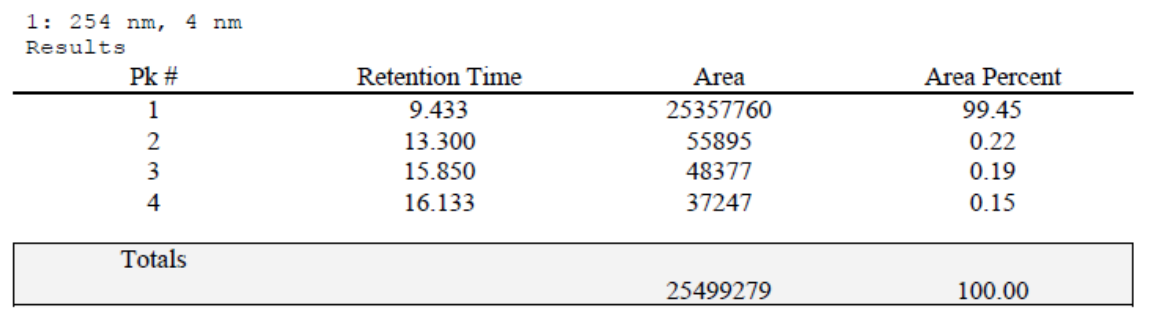


Figure S12. HPLC chromatograms for 4-amino-3-chloro-6-(4-chloro-2-fluoro-3methoxyphenyl)-5-fluoro(4- $\left.{ }^{14} \mathrm{C}\right)$ pyridine-2-carbaldehyde (28).
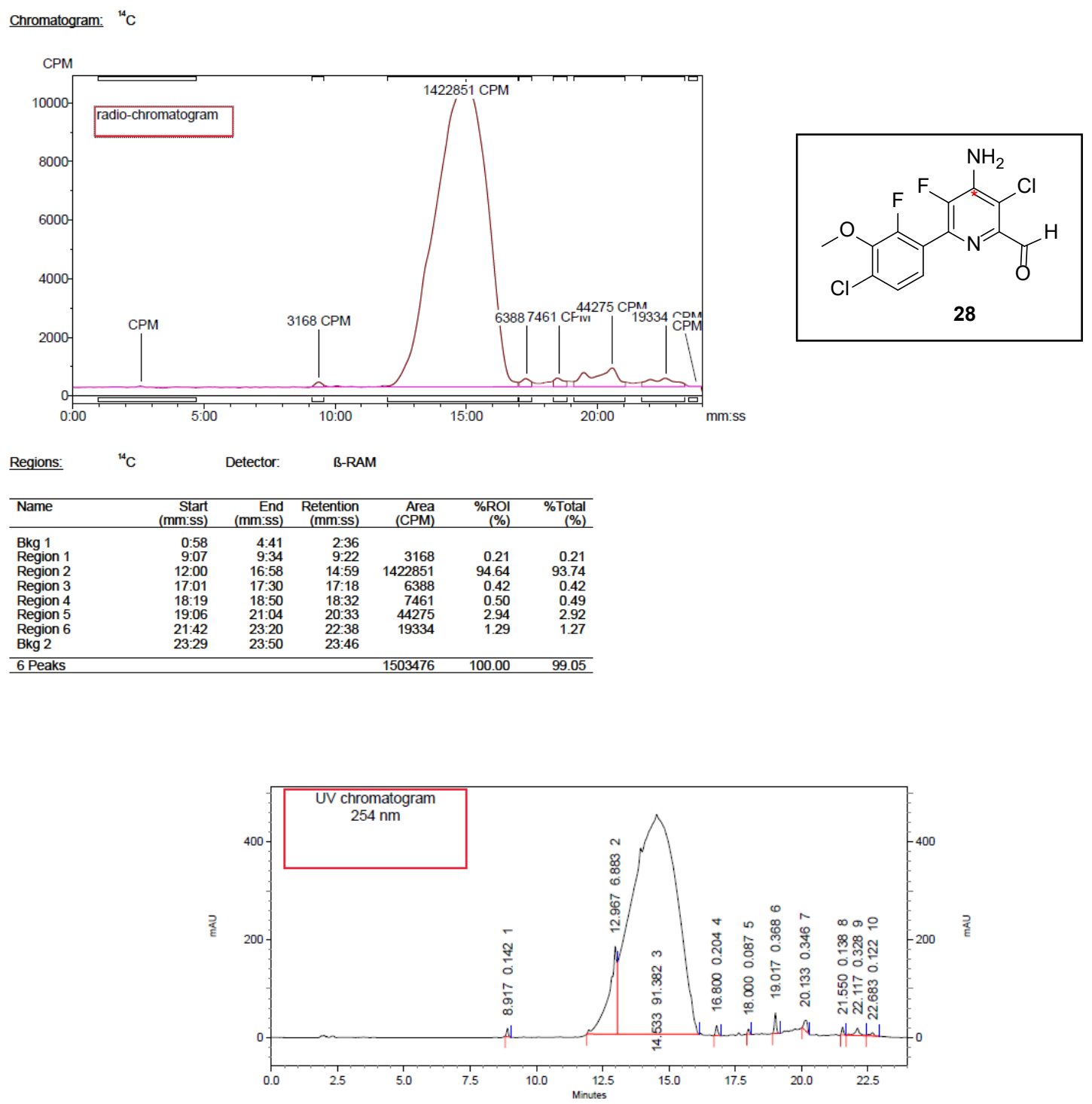

\begin{tabular}{lccc}
$\begin{array}{l}\text { 1: } 254 \mathrm{~nm}, 4 \mathrm{~nm} \\
\text { Results }\end{array}$ & & \\
$\mathrm{Pk} \#$ & Retention Time & Area & Area Percent \\
\hline 1 & 8.917 & 81290 & 0.14 \\
2 & 12.967 & 3938098 & 6.88 \\
3 & 14.533 & 52285977 & 91.38 \\
4 & 16.800 & 116819 & 0.20 \\
5 & 18.000 & 49776 & 0.09 \\
6 & 19.017 & 210318 & 0.37 \\
7 & 20.133 & 198113 & 0.35 \\
8 & 21.550 & 78918 & 0.14 \\
9 & 22.117 & 187787 & 0.33 \\
10 & 22.683 & 69587 & 0.12 \\
\hline Totals & & \\
\end{tabular}


Figure S13. HPLC chromatograms for 4-amino-3-chloro-6-(4-chloro-2-fluoro-3methoxyphenyl)-5-fluoro( $\left(4-{ }^{14} \mathrm{C}\right)$ pyridine-2-carboxylic acid (29).
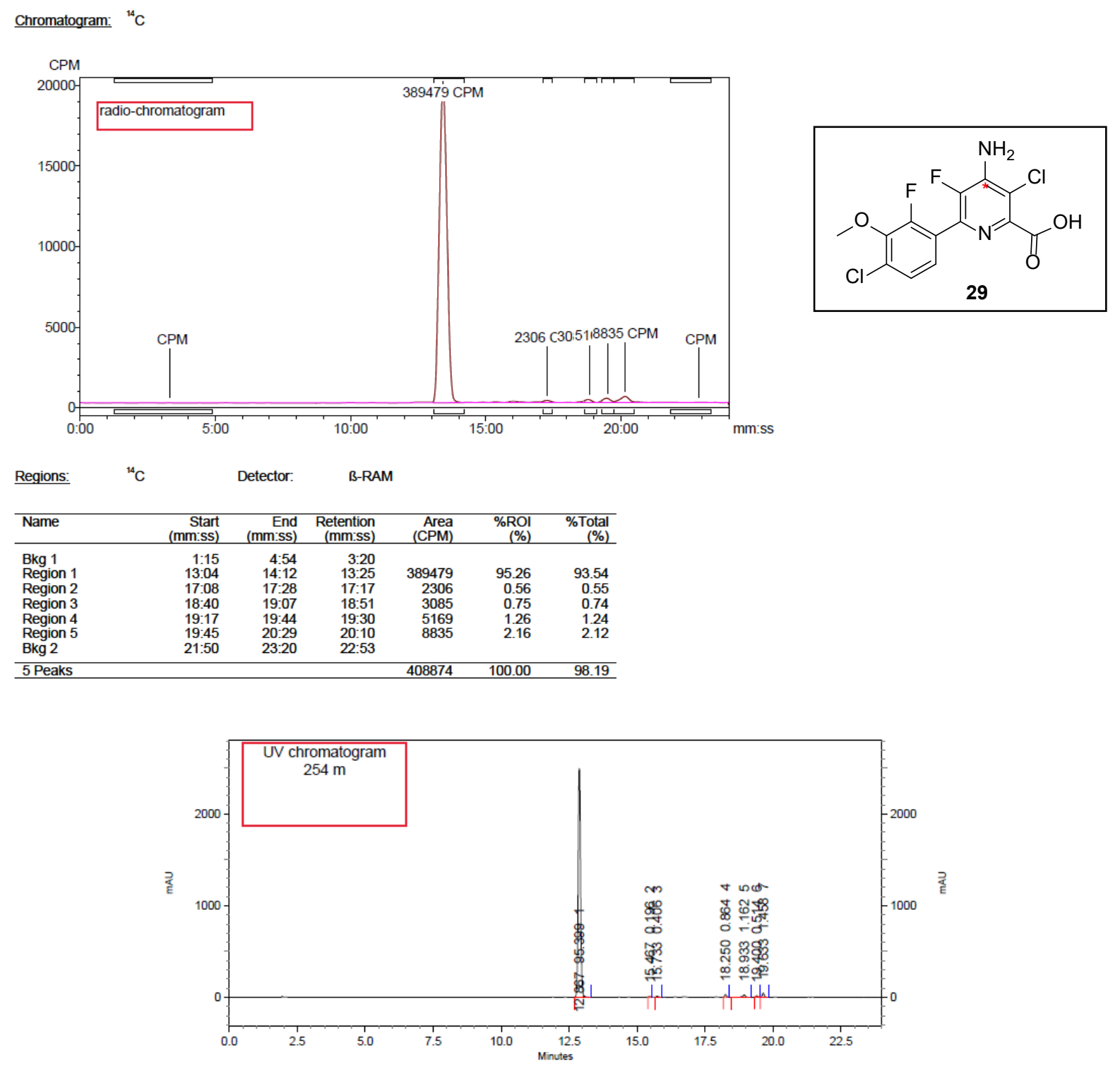

\begin{tabular}{rccc}
$\begin{array}{l}\text { 1: } 254 \mathrm{~nm}, 4 \mathrm{~nm} \\
\text { Results }\end{array}$ & & \\
Pk \# & Retention Time & Area & Area Percent \\
\hline 1 & 12.867 & 15443992 & 95.40 \\
2 & 15.467 & 31717 & 0.20 \\
3 & 15.733 & 65654 & 0.41 \\
4 & 18.250 & 139917 & 0.86 \\
5 & 18.933 & 188141 & 1.16 \\
6 & 19.400 & 83258 & 0.51 \\
7 & 19.633 & 236082 & 1.46 \\
\hline Totals & & \\
\hline
\end{tabular}


Figure S14. HPLC chromatograms for benzyl 4-amino-3-chloro-6-(4-chloro-2-fluoro-3methoxyphenyl)-5-fluoro( $\left(4-{ }^{14} \mathrm{C}\right)$ pyridine-2-carboxylate $\left(30\right.$, Rinskor-Py-4- $\left.{ }^{14} \mathrm{C}\right)$.
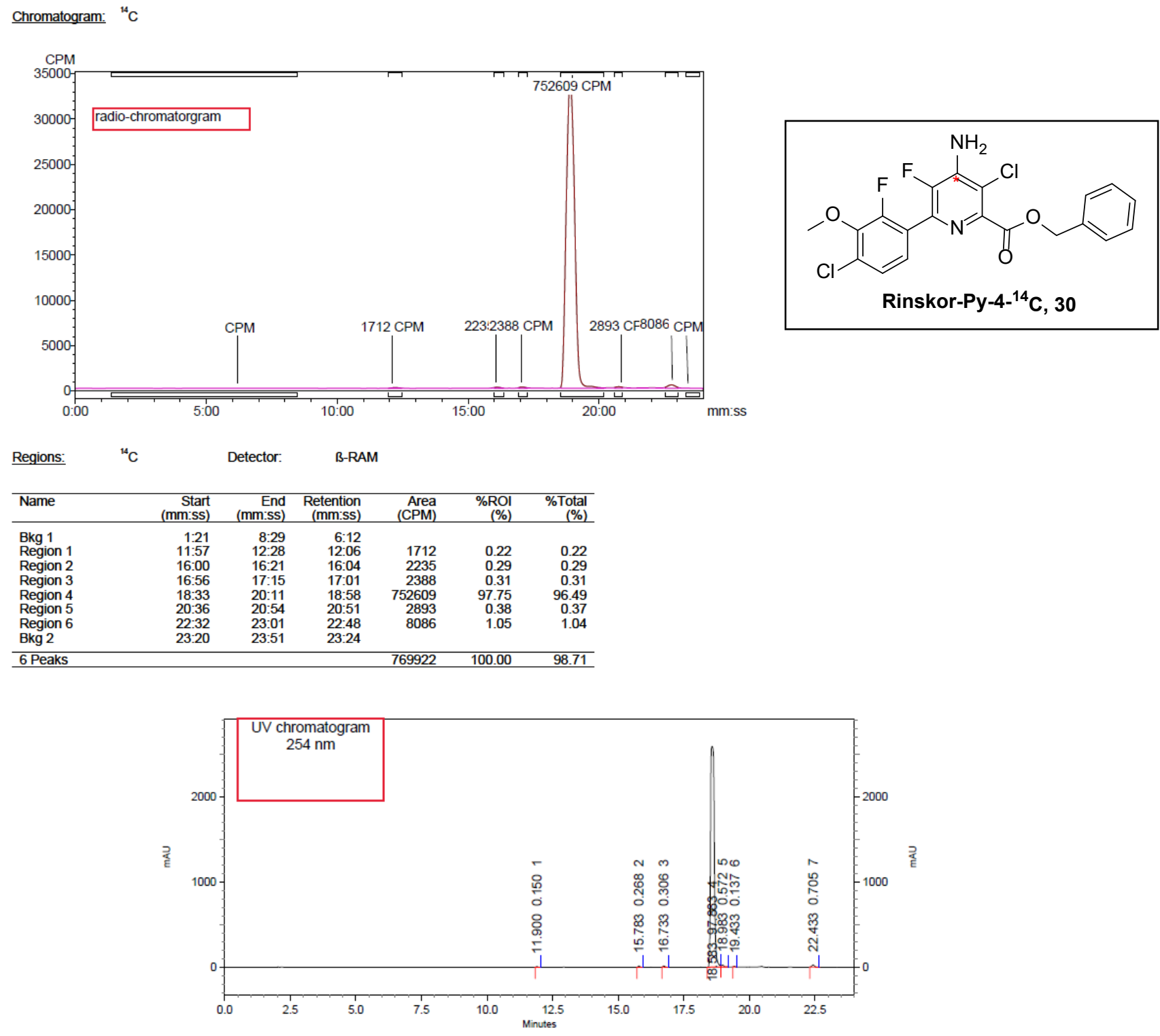

1: $254 \mathrm{~nm}, 4 \mathrm{~nm}$

Results

\begin{tabular}{cccc} 
Pk \# & Retention Time & Area & Area Percent \\
\hline 1 & 11.900 & 38738 & 0.15 \\
2 & 15.783 & 69118 & 0.27 \\
3 & 16.733 & 78986 & 0.31 \\
4 & 18.583 & 25267155 & 97.86 \\
5 & 18.983 & 147641 & 0.57 \\
6 & 19.433 & 35326 & 0.14 \\
7 & 22.433 & 181917 & 0.70
\end{tabular}

Totals

25818881

100.00 